\title{
Origin and hydrothermal alteration of rare-metal granites in the Al-Hamra area, northeastern Arabian Shield, Saudi Arabia
}

Talal M. Qadhi

Department of M ineral Resources and Rocks,

Faculty of Earth Sciences, King A bdulaziz U niversity, Jeddah

The Jabal Hamra (538 Ma) and Jabal Abu ad Dud plutons in northeast Saudi Arabia are epizonal bodies consisting of alkali feldspar granite and alkali feldspar syenite. Fracture-controlled zones of highly altered granites are recorded along the margins of the plutons. The granites intrude metamorphosed volcano-sedimentary successions of the Matran Formation. The rocks of the two plutons are chemically indistinguishable. They are characterized by above-average $\mathrm{Th}, \mathrm{Nb}, \mathrm{Y}, \mathrm{Ta}, \mathrm{Hf}$ and $\mathrm{Zr}$, very low $\mathrm{CaO}, \mathrm{TiO}_{2}, \mathrm{MgO}, \mathrm{FeO}$ and $\mathrm{MnO}$, and by high contents of rare earth elements (REE). Tectonic discrimination diagrams suggest an intra-plate environment, with many geochemical and mineralogical features resembling post-orogenic A-type granites. Numerous local and regional geologic constraints indicate that the plutons were intruded in an extension-related setting following the cessation of Neoproterozoic arc-related magmatism. Geochemical data are consistent with their derivation by partial melting of depleted crust followed by fractional crystallization of feldspars, ferromagnesian minerals and REE-rich accessory phases. The radiogenic isotope data [eN $d(T)$ values are +3.5 to +4.2 ] indicate that the granite magma was generated from a 'juvenile' source, which is typical of the rocks making up most of the Arabian-Nubian Shield. Rare-metal mineralization is associated with the fracture-controlled alteration zones that occur at Jabal Al Hamra and Jabal Abu ad Dud. The altered rocks are characterized by higher $\mathrm{TiO}_{2}, \mathrm{Fe}_{2} \mathrm{O}_{3}, \mathrm{SiO}_{2}$ and lower $\mathrm{Al}_{2} \mathrm{O}_{3}, \mathrm{CaO}, \mathrm{Na}_{2} \mathrm{O}$, than the unaltered rocks. They show high and wide range in the total REE contents (804-15579 ppm), Ta (6-194 ppm), Nb (51-3483 ppm), Hf (13-368 ppm), Zr (394-14887 ppm), Th (16-572 ppm) and U (4-143 ppm). Field observations and further petrographic and chemical studies suggest that the altered rocks and the rare metal enrichment are the products of hydrothermal-metasomatic alteration of the quartz alkali feldspar syenite and the alkali feldspar granite. The rare-metal enrichment was explained by orthomagmatic fluid transport of these elements as fluoride complexes, and their subsequent deposition as a result of mixing with externally derived Fe-rich fluids.

Key words: granite, rare earth elements, Arabian Shield, fracture-controlled alteration zone

Address: T. M. Qadhi: 80206Jeddah, Saudi Arabia, e-mail: tquadi@kau.edu.sa

Received: March 10, 2007; accepted: July 2, 2007 


\section{Introduction}

The Arabian Shield is the exposed Precambrian basement region of the Arabian Peninsula of Saudi Arabia, Yemen and Oman, with the bulk of the exposed Precambrian lying within western Saudi Arabia. It is part of the Arabian-Nubian Shield (ANS) that also includes northeastern Africa, Sinai and Jordan. The ANS comprises a vast expanse of Neoproterozoic juvenile oceanic island arc crust, flanked on the west and east by older cratonic crust. The ANS, which represents one of the largest tracts of juvenile crust on Earth, occupies the northern part of the East African Orogen (EAO) of Stern (1994, 2002), a major Neoproterozoic orogen that resulted from the collision of the East and West Gondwana continents to form the Gondwana super-continent near the end of the Neoproterozoic.

Among all geologic environments, alkaline igneous settings are the most enriched in HFSE and are commonly potential repositories of deposits of these metals. Economic or potentially economic HFSE concentrations hosted in alkaline igneous rocks have been interpreted either due to fractional crystallization or hydrothermal alteration (Schwart 1992). The Arabian Shield contains one of the largest fields of alkali granites in the world. It constitutes about $2.3 \%$ of the plutonic rock assemblage in the Arabian Shield (Stoeser 1986). They form, along with the some aluminous granite, one of the economically important groups of granites in the shield and are associated with $\mathrm{Nb}, \mathrm{Ta}$, Th, U, Sn, W, F, Mo, Zr, Y and REE mineralization (Drysdall et al. 1984; Ramsay et al. 1986; Jackson 1986; Q adhi 1990). Forty-nine major and more than a dozen minor alkaline and peralkaline granite plutons are concentrated in the Midyan and Hijaz terrains and in the Nabitah orogenic belt. Very few alkali granite plutons are defined in the southern part of the shield. In general, alkali granite is the last major intrusive phase wherever it occurs. Available radiometric data show that the alkali granite was emplaced during a time span of about $180 \mathrm{Ma}(686-518 \mathrm{Ma})$; all but four occurrences formed between 630-565 Ma. A major part of the alkaline/peralkaline granites have chemical specialization characters (Tischendorf 1977; Du Bray 1986; Ramsay 1986) and some show marked enrichment in Nb, Ta, Th, U, Zr, Y and REE.

The Hamra area (Fig. 1), in northeastern Saudi Arabia, contains two rare-metalbearing granite plutons (Jabal Al Hamra and Jabal Abu ad Dud), which were affected by hydrothermal alteration. The plutons are made up of alkali-feldspar syenite and alkali-feldspar granite. In this paper we present geologic, mineralogical, chemical and radiogenic isotope data, to constrain the likely processes responsible for the origin of the granite and the associated mineralization.

\section{Field geology and petrography}

The Jabal Al Hamra area is located between lat. $26^{\circ}$ and $27^{\circ} \mathrm{N}$ and long. $37^{\circ} 30$ and $39^{\circ} 00 \mathrm{E}$. The geologic map (Fig. 2) shows that the intrusive plutonic rocks 
Fig. 1

Location of Al Hamra area (black point), Arabian Shield. Saudi Arabia

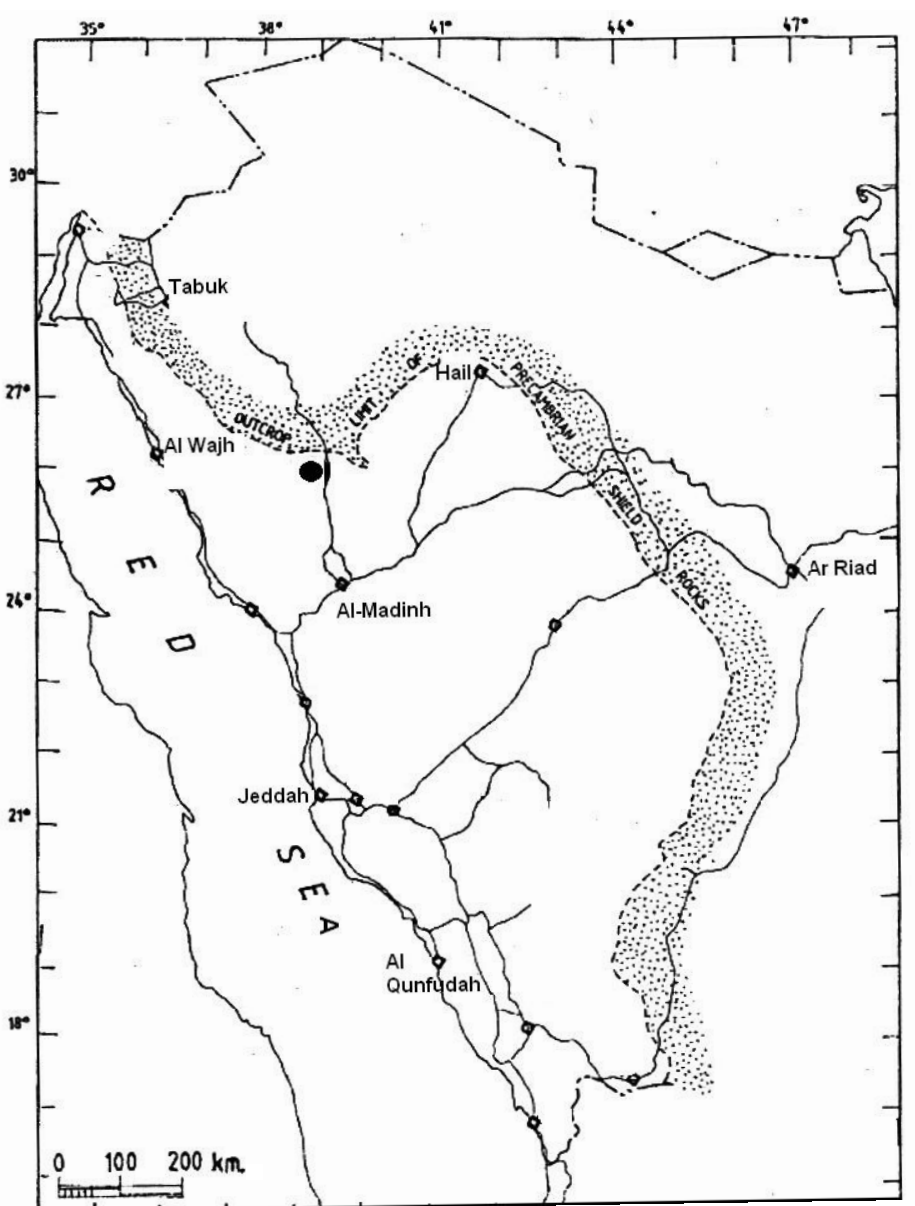

include Jabal Al Hamra, Jabal Abu ad Dud and Jabal Sinn, which were intruded into a metamorphosed volcano-sedimentary succession of the Matran Formation. These intrusive rocks are unconformably overlain by younger volcanic and sedimentary rocks of the Rubtayn Formation of the Jibalah Group (Hadley 1975). The rocks of Sahl al Matran evolved in a late Neoproterozoic island arc (Schmidt et al. 1979; Greenwood et al. 1979; Fleck et al. 1980), approximately between 900 and $600 \mathrm{Ma}$ ago. This period was followed by a late N eoproterozoic post-collision event ( $\mathrm{N}$ ajd Orogeny) during which the $\mathrm{N}$ ajd fault system was established, the Jibalah Group was deposited and deformed and the intrusive alkaline rocks were intruded (Schmidt et al. 1979; Fleck et al. 1980; Kemp et al. 1980). 


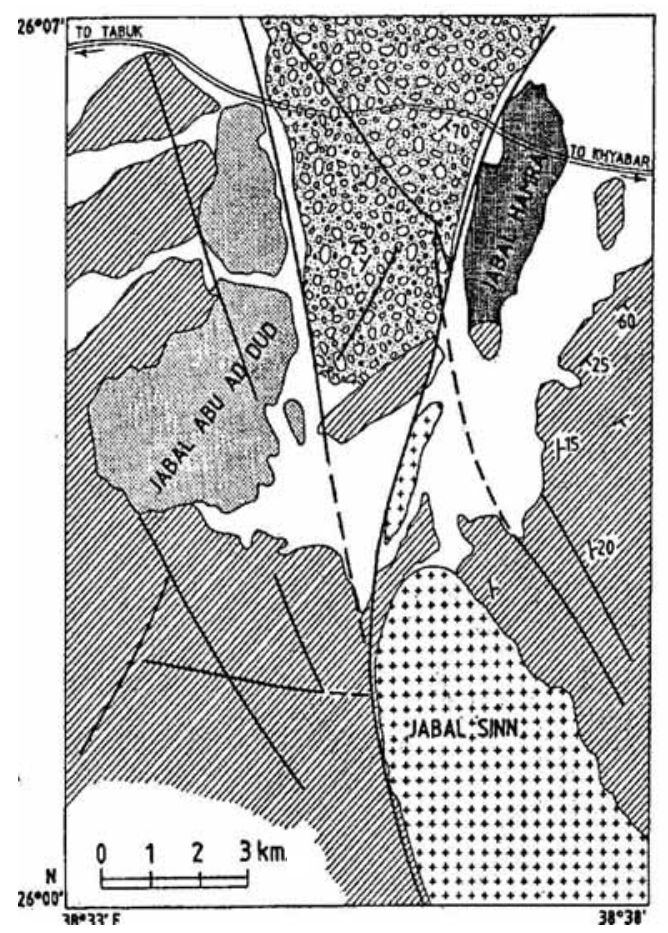

$\square$ Wadi deposits, sands and pediment Alluvium

60.0 Jibalah group (Volcanic and sedimentary eies rocks)

(5) Quartz syenite, microgranite (Jabal Al Hamra)

Syenite, alkall feldspar granite and quartz 1.8 monzonite (Jabal Abu ad Dud)

7.7. Microgranite, monzogranite and alkal ¿.:.1 feldspar granite (Jabal Sinn)

Martan Formation (AI Ays group)

- Faults dashed when inferred

$\lambda$ Strike and dip of inclined beds

Fig. 2

Geologic map of Al Hamra area (modified after Hadley 1987)

Jabal Al H amra

The Jabal Al Hamra is a 5 by $1.3 \mathrm{~km} \mathrm{~N}$-trending ridge (Fig. 2) rising approximately $250 \mathrm{~m}$ above wadi level. Marginal tectonic brecciation and pervasive cataclasis are recorded, particularly at the western side of the granite mass, indicating a forceful mode of emplacement (Drysdall et al. 1984). The rocks of the Hamra pluton are massive, homogeneous and fine to medium-grained. Some parts of the pluton show a variable degree of deformation and hydrothermal alteration that are more common and intense in the southern part of the pluton. The pluton consists of reddish quartz alkali-feldspar syenite, whereas coarsegrained alkali-feldspar granite predominates in the northern part of the pluton. Xenoliths of the country rock (Matran Formation) are recorded in the quartz alkali-feldspar syenite. They are of blackish grey color, sub-rounded to irregular and highly sheared.

The quartz alkali-feldspar syenite constitutes the major part of Jabal Hamra. The rocks are equigranular, fine to medium-grained, of pink to red color. Some samples show porphyritic texture, especially in the roof facies. The rocks consist of alkali feldspar, quartz (constituting up to 90 to $95 \%$ of the rock mode) and minor amounts of fine albite laths. Mafic minerals are partially to completely 
replaced by iron oxides, quartz and calcite. Accessory minerals include zircon, fluorite and allanite. Alkali-feldspar is represented by microcline and microclineperthite (about 75 to $85 \%$ of the rock mode). Some crystals of alkali feldspar are invariably clouded and stained by pale reddish brown materials (a mixture of iron oxides and clay minerals). They also exhibit clear colorless dentate al bite rims and veinlets. Quart crystals show wide variation in size and abundance (10 to $25 \%$ of the rock mode); there is therefore a local gradation from quart alkalifeldspar syenite to alkali-feldspar granite. The quartz crystals are mediumgrained with an anhedral granular form. Secondary quart of post-magmatic origin occurs in very fine-grained micro- to cryptocrystalline aggregates, almost forming veinlets.

The alkali-feldspar granite is petrographically and mineralogically similar to the quartz alkali-feldspar syenite, but the former is of limited distribution in Jabal Hamra. Moreover, it differs by the high modal content of quartz that exceeds $20 \%$ of the rock mode. Silicification is pronounced in some samples. K-feldspar and quartz are the main constituents of the rock with subordinate plagioclase feldspar. Secondary minerals are abundant in some samples and include quartz, calcite and iron oxides.

The altered alkali feldspar syenite represents a highly silicified, hematitized and fracture-controlled zone $(300 \times 100 \mathrm{~m})$ on the southern side of the Hamra granite pluton. The rocks in this zone are very fine-grained to porphyritic and of dark color, ranging from black and dark brown to deep reddish-brown, with abundant opaque minerals. High radioactivity (up to $4000 \mathrm{cps}$; average background is $250 \mathrm{cps}$ ) is recorded in the altered granite zone. The altered zone is in irregular and gradational contact against the adjacent quartz alkali-feldspar syenite. Jackson and Douch (1986) recorded a sharp, brecciated and sheared contact between the altered granite and the surrounding quartz alkali-feldspar syenite. However, field observations and further petrographic and chemical studies suggest that the altered granite is not magmatic rock but a type of hydrothermal alteration. The rocks of the altered zone are fine-grained, of yellowishbrown to reddish-brown and dark black color. It is heterogeneous, with the main phenocrysts being quartz. Cluster aggregates of recrystallized fine-grained quartz form a glomeroporhyrtic-like texture. The medium-grained quartz crystals ( $4 \mathrm{~mm}$ average diameter) are highly strained, corroded and embayed. The matrix consists of very fine-grained, micro- to crypto-crystalline quartz admixed with yellowish-brown to deep brown hematite, hydrated iron oxides, fluorite, zircon, sericite and calcite. Relicts of fine-grained, highly altered alkali-feldspar are also found in the matrix.

Jabal A bu ad Dud

The Jabal Abu ad Dud (Fig. 2) forms a north-trending, elongated pluton of pink, reddish to brick red alkali feldspar granite. The rocks are massive to locally foliated, homogenous and medium- to coarse-grained. They show variable 
degrees of shearing and deformation. However, the deformation features are prominent in the northern part of the pluton, while the southern part is less deformed. A field radiometric survey over the Abu ad Dud granite mass, particularly in the northern part of the pluton, reveals local high radioactivity with up to $2500 \mathrm{cps}$. Alteration zones and patches of silica-hematite-rich granite are recorded in Jabal Abu ad Dud.

The alkali feldspar granite constitutes the main part of Jabal Abu ad Dud pluton, although minor porphyritic microgranite is also recorded. The rocks are coarse-grained, hypersolvous and equigranular of hypidiomorphic texture. Local deformation and shearing are prominent. The rocks consist of K-feldspar and quartz. The mafic minerals are almost altered to iron oxides that impart the reddish-brown coloration to the rock. K-feldspar (orthoclase) crystals are mostly flame perthites and mesoperthites forming 60 to $80 \%$ of the rock mode. The crystals are coarse-grained and subhedral to anhedral. Some crystals are highly clouded and turbid due to alteration, and often with a clear albite rim. Quartz shows noticeable variation in abundance, ranging from $10 \%$ to $30 \%$ of the rock mode. Two types of quartz crystals are identified in the rock. The first type is coarse- to medium-grained, anhedral, of granular form and with corroded grain boundaries. Some quartz crystals contain poikilitic inclusions of perthites; most of them are highly strained as manifested by severe undulose extinction. The second type occurs as very fine-grained to cryptocrystalline aggregates. They are anhedral, undeformed and commonly admixed with iron oxides. Accessory minerals include zircon, thorite, rutile and monazite.

The altered rocks in the Abu ad Dud pluton are mineralogically and chemically very similar to those observed at Jabal Hamra.

\section{Analytical techniques}

Based on the petrographic investigations, 31 representative samples covering the different granite varieties were selected for major and trace element analyses. Major element compositions and Sc, Ba and $\mathrm{Ni}$ abundances were determined by inductively coupled plasma-atomic emission spectrometry (ICP-AES). The remainder of trace elements and the rare earth elements (REE) were determined by inductively coupled plasma-mass spectrometry (ICP-MS). All the analyses were carried out at the ACME analytical laboratories Ltd., Canada. Analytical precision, as calculated from replicate analyses, is $0.5 \%$ for major elements and varies from $2-20 \%$ for trace elements. The major and trace element data of the Jabal Hamra and Jabal Abu ad Dud are given in Table 1. Isotopic ratios of $\mathrm{Sr}$ and $\mathrm{Nd}$, and the concentrations of $\mathrm{Rb}, \mathrm{Sr}, \mathrm{Sm}$ and $\mathrm{Nd}$, were determined by isotopic dilution analysis. The analyses were undertaken at the Geology Department, Bergen University (Norway), using a VG 354 and Finnigan MAT 262 mass spectrometer. The ${ }^{87} \mathrm{Sr} /{ }^{66} \mathrm{Sr}$ and the ${ }^{143} \mathrm{~N} \mathrm{~d} /{ }^{144} \mathrm{~N} \mathrm{~d}$ ratios were normalized within runs to ${ }^{87} \mathrm{Sr} /{ }^{86} \mathrm{Sr}=0.1194$ and to ${ }^{146} \mathrm{Nd} /{ }^{144} \mathrm{Nd}=0.7219$. Laboratory values for 
standards at the time of running the samples were: Johanson and Matthey (JM) $\mathrm{Nd}_{2} \mathrm{O}_{3}$, batch no. S819093A yielded ${ }^{143} \mathrm{~N} \mathrm{~d} /{ }^{144} \mathrm{Nd}=0.511101 \pm 15(2 \mathrm{~s}) ; \mathrm{NBS} 987$ yielded ${ }^{87} \mathrm{Sr} / 86 \mathrm{Sr}=0.71015 \pm 0.00004$ ( $2 \sigma$, mean). The decay constant used for ${ }^{147} \mathrm{Sm}$ is $6.54 \times 10^{-12} \mathrm{y}^{-1}$ and for ${ }^{87} \mathrm{Rb}$ is $1.42 \times 10^{-11} \mathrm{y}^{-1}$ (Steiger and Jager 1977). Model $\mathrm{Nd}$ ages $\left(\mathrm{T}_{\mathrm{DM}}\right)$ were calculated according to the depleted mantle model of DePaolo (1981).

\section{Chemical variations in the granites}

The studied rocks are classified using the R1-R2 diagram (Fig. 3a) of De La Roche et al. (1980) and of Batchelor and Bowden (1985). All the investigated samples plot in the field of alkali granites and follow the alkaline trend. Most of the unaltered rocks of Jabal Hamra and Jabal Abu ad Dud are metaluminous to peralkaline (Fig. 3b), except two samples ( $\mathrm{H}-11$ and $\mathrm{H}-16)$, which are peraluminous. The Jabal Hamra and Jabal Abu ad Dud granites show a wide variation and enrichment in term of most major and trace elements $\left(\mathrm{SiO}_{2}\right.$ $=58.7-74.9$ wt\%; $\mathrm{Al}_{2} \mathrm{O}_{3}=$ 9.5-15.5 wt\%; $\mathrm{Ba}=95-624$ ppm; $\mathrm{Zr}=200-6000 \mathrm{ppm} ; \mathrm{Nb}=$ 30-1000 ppm; Th = 3-100 ppm). The major element variation diagrams (Fig. 4) show the following: 1) The rocks of the Jabal Hamra and Abu ad Dud display similar behavior in most of their major and trace element trends. 2) $\mathrm{TiO}_{2}, \mathrm{CaO}, \mathrm{Al}_{2} \mathrm{O}_{3}$, and total $\mathrm{Fe}$ as $\mathrm{Fe}_{2} \mathrm{O}_{3}$ decrease with increasing $\mathrm{SiO}_{2}$. Zirconium, Th and $\mathrm{Nb}$ contents increase with increasing $\mathrm{Rb}$ (Fig. 5). Although data points are rather scattered, the alteration product of the quartz alkali feldspar syenite of Jabal Hamra and the alkali

Fig. 3

a) R1-R2 diagram (De La Roche et al. 1980), b) A plot of Shand index (A/CNK vs. A/NK) for Al Hamra granites; discrimination fields are from Maniar and Piccoli 1989)

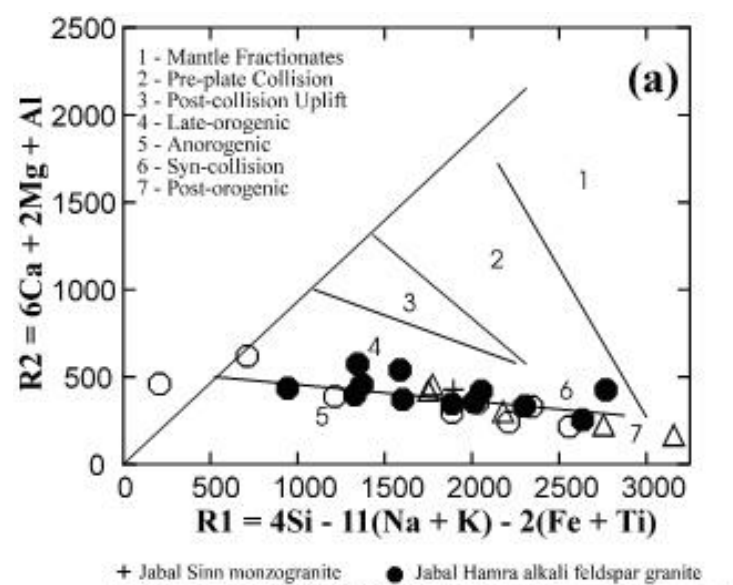

O Jabal Sinn monzogranite
O Jabal Abu ad Dud alkali feldspar granite $\Delta$ hydrothermally altered rocks

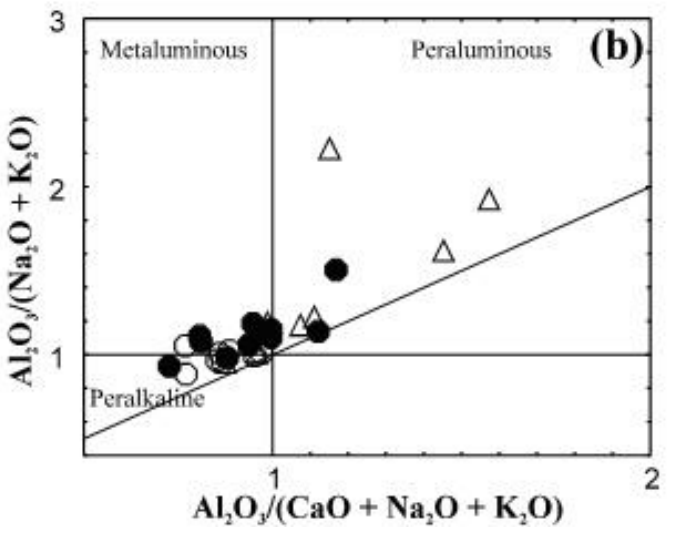

Central European Geology 50, 2007 


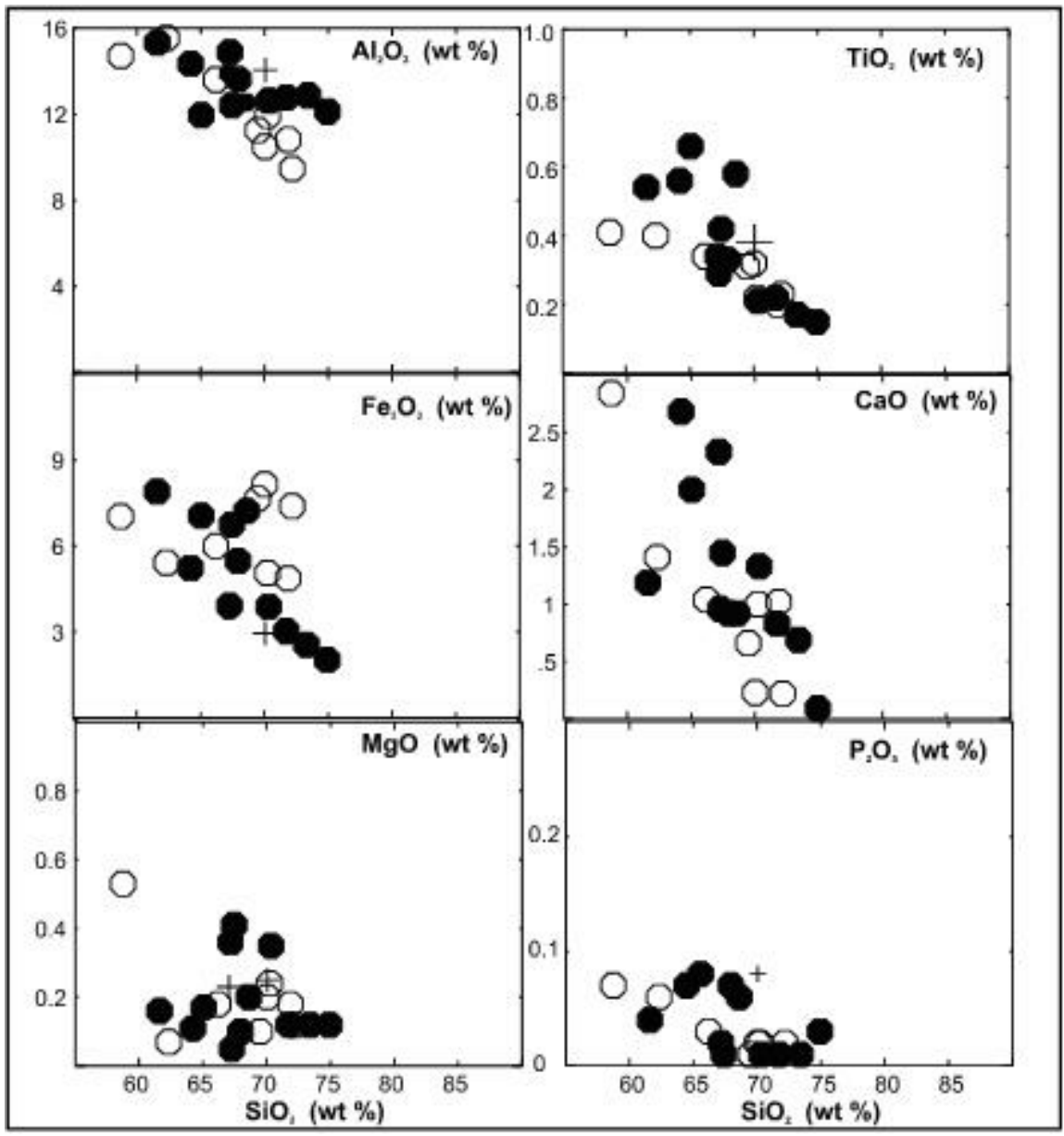

Fig. 4

Harker variation diagrams of some major of the Jabal Al Hamra, Jabal Abu ad Dod and Jabal Sinn unaltered granites. Symbols as in Fig. 3

feldspar granite of Jabal Abu ad Dud are characterized by higher contents in $\mathrm{TiO}_{2}, \mathrm{Fe}_{2} \mathrm{O}_{3}, \mathrm{SiO}_{2}, \mathrm{Ta}, \mathrm{Nb}, \mathrm{Hf}, \mathrm{Zr}$, Th and $\mathrm{U}$ and lower in $\mathrm{Al}_{2} \mathrm{O}_{3}, \mathrm{CaO}, \mathrm{Na}_{2} \mathrm{O}$, than the unaltered rocks (Table 1 and Figs 4 and 5).

The variation of trace elements is well demonstrated on primordial mantlenormalized diagrams using the normalizing mantle values of Sun (1982). All the investigated rocks from the Jabal Hamra and Jabal Abu ad Dud show similar variation patterns and element enrichment and depletion (Fig. 6). This may indicate that they evolved from the same source and/or comparable fractionation history. Well-defined negative anomalies are observed for $\mathrm{Sr}, \mathrm{Ba}, \mathrm{K}$ and $\mathrm{Ti}$ among 


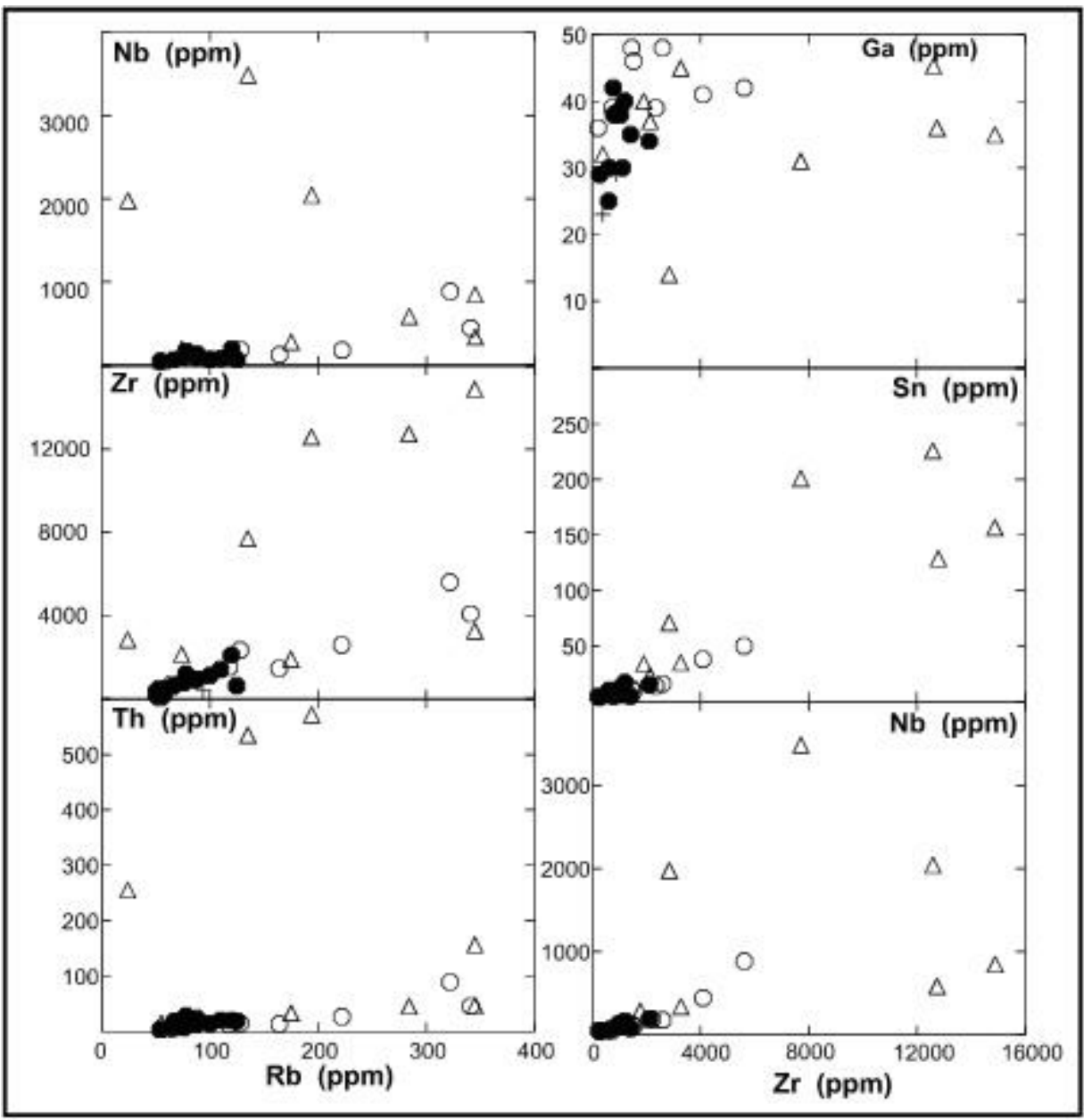

Fig. 5

Trace element variation diagrams of the Jabal Al Hamra, Jabal Abu ad Dod and Jabal Sinn unaltered granites. Symbols as in Fig. 3

all the rocks that are attributed to the fractionation of plagioclase (Sr), K-feldspar $(\mathrm{K}, \mathrm{Ba})$ and Fe-Ti oxides (Ti). The syenogranite of Jabal Sinn shows enrichment in large-ion lithophile elements (LILE) and distinctive depletion in Nb (Fig. 6), which is a characteristic feature of arc granitoids.

Rare earth elements of the studied rocks from Jabal Hamra and Abu ad Dud are manifested in chondrite-normalized diagrams (Fig. 7). The alkali feldspar syenite of Jabal Hamra and the alkali feldspar granite of Jabal Abu ad Dud show very similar REE patterns. They show slight differences in the intensity of the Eu anomalies and total REE. The quartz alkali feldspar syenite samples are char- 
268 T. M. Q adhi

Table 1

Chemical data of Al Hamra and Abu ad Dod alkali feldspar granites (AFG)

\begin{tabular}{|c|c|c|c|c|c|c|c|c|c|c|c|}
\hline \multicolumn{12}{|c|}{ Al Hamra AFG } \\
\hline Sample & H-18 & H-20 & H-1 & H-2 & H-10A & H-11 & H-12A & H-13 & H-14 & H-15 & H-16 \\
\hline $\mathrm{SiO}_{2}$ & 67.05 & 70.05 & 74.92 & 65.05 & 71.72 & 67.27 & 67.46 & 70.33 & 64.21 & 67.16 & 61.60 \\
\hline $\mathrm{TiO}_{2}$ & 0.28 & 0.38 & 0.15 & 0.66 & 0.22 & 0.29 & 0.42 & 0.21 & 0.56 & 0.34 & 0.54 \\
\hline $\mathrm{Al}_{2} \mathrm{O}_{3}$ & 14.86 & 14.05 & 12.12 & 11.96 & 12.78 & 14.88 & 12.42 & 12.63 & 14.34 & 13.89 & 15.34 \\
\hline $\mathrm{Fe}_{2} \mathrm{O}_{3}$ & 3.85 & 2.96 & 2.02 & 7.06 & 3.03 & 3.92 & 6.75 & 3.88 & 5.23 & 3.77 & 7.90 \\
\hline $\mathrm{MnO}$ & 0.09 & 0.06 & 0.01 & 0.23 & 0.04 & 0.11 & 0.10 & 0.09 & 0.09 & 0.09 & 0.24 \\
\hline $\mathrm{MgO}$ & 0.23 & 0.25 & 0.12 & 0.17 & 0.12 & 0.05 & 0.41 & 0.35 & 0.11 & 0.36 & 0.16 \\
\hline $\mathrm{CaO}$ & 0.95 & 1.30 & 0.09 & 2.00 & 0.83 & 0.96 & 1.45 & 1.33 & 2.68 & 2.33 & 1.19 \\
\hline $\mathrm{Na}_{2} \mathrm{O}$ & 4.84 & 4.70 & 3.72 & 5.18 & 4.66 & 5.18 & 3.25 & 1.78 & 4.74 & 4.75 & 5.00 \\
\hline $\mathrm{K}_{2} \mathrm{O}$ & 5.69 & 4.36 & 4.19 & 3.98 & 4.94 & 5.17 & 4.73 & 5.05 & 4.72 & 4.69 & 5.02 \\
\hline $\mathrm{P}_{2} \mathrm{O}_{5}$ & 0.01 & 0.08 & 0.03 & 0.08 & 0.01 & 0.01 & 0.01 & 0.01 & 0.07 & 0.02 & 0.04 \\
\hline LOI & 0.72 & 0.65 & 1.98 & 2.67 & 1.23 & 1.56 & 2.44 & 3.87 & 2.87 & 2.54 & 2.11 \\
\hline Sum & 98.57 & 98.84 & 99.35 & 99.04 & 99.58 & 99.40 & 99.44 & 99.53 & 99.62 & 99.94 & 99.14 \\
\hline $\mathrm{Ni}$ & 1 & 2 & 2 & 3 & 2 & 2 & 6 & 1 & 1 & 2 & 1 \\
\hline $\mathrm{Co}$ & 1 & 4 & 6 & 2 & 4 & 1 & 3 & 2 & 1 & 2 & 1 \\
\hline $\mathrm{Sc}$ & 3 & 4 & 1 & 14 & 2 & 2 & 2 & 2 & 8 & 4 & 1 \\
\hline V & 3 & 15 & 3 & 3 & 3 & 3 & 12 & 3 & 3 & 5 & 3 \\
\hline $\mathrm{Cu}$ & 3 & 4 & 19 & 10 & 59 & 3 & 17 & 5 & 15 & 19 & 6 \\
\hline $\mathrm{Pb}$ & 4 & 11 & 11 & 26 & 4 & 4 & 13 & 4 & 11 & 6 & 4 \\
\hline $\mathrm{Zn}$ & 115 & 71 & 10 & 119 & 28 & 122 & 574 & 61 & 18 & 61 & 233 \\
\hline $\mathrm{Sn}$ & 2 & 5 & 7 & 4 & 5 & 5 & 15 & 5 & 7 & 6 & 7 \\
\hline $\mathrm{W}$ & 11 & 22 & 133 & 23 & 69 & 21 & 23 & 33 & 3 & 20 & 14 \\
\hline $\mathrm{Rb}$ & 77 & 94 & 67 & 54 & 88 & 87 & 120 & 110 & 66 & 100 & 76 \\
\hline Cs & 0.4 & 1.1 & 0.7 & 0.6 & 0.5 & 0.8 & 0.9 & 1.2 & 1.4 & 0.8 & 1.2 \\
\hline $\mathrm{Ba}$ & 75 & 426 & 115 & 750 & 138 & 114 & 264 & 109 & 270 & 157 & 178 \\
\hline $\mathrm{Sr}$ & 17 & 107 & 19 & 49 & 20 & 22 & 48 & 25 & 54 & 33 & 35 \\
\hline $\mathrm{Ga}$ & 29 & 23 & 30 & 29 & 38 & 38 & 34 & 35 & 25 & 30 & 42 \\
\hline $\mathrm{Ta}$ & 2.4 & 3.1 & 9.5 & 3.0 & 6.9 & 5.5 & 16.8 & 10.1 & 4.3 & 5.6 & 5.9 \\
\hline $\mathrm{Nb}$ & 39 & 31 & 63 & 48 & 62 & 59 & 198 & 80 & 65 & 70 & 71 \\
\hline Hf & 16 & 12 & 19 & 7 & 26 & 22 & 45 & 36 & 14 & 26 & 17 \\
\hline $\mathrm{Zr}$ & 879 & 396 & 558 & 281 & 1055 & 797 & 2110 & 1421 & 616 & 1123 & 784 \\
\hline $\mathrm{Y}$ & 51 & 44 & 87 & 50 & 90 & 83 & 188 & 139 & 62 & 94 & 80 \\
\hline $\mathrm{Th}$ & 5.9 & 13.7 & 19.0 & 4.3 & 12.3 & 12.5 & 20.4 & 21.0 & 8.7 & 14.7 & 7.4 \\
\hline $\mathrm{U}$ & 3.0 & 5.1 & 3.7 & 2.4 & 5.4 & 4.6 & 10.0 & 6.7 & 2.8 & 5.5 & 4.5 \\
\hline $\mathrm{La}$ & 79.40 & 46.00 & 50.30 & 39.40 & 75.00 & 47.20 & 203.90 & 111.30 & 61.00 & 101.70 & 83.20 \\
\hline $\mathrm{Ce}$ & 169.60 & 98.60 & 118.00 & 91.20 & 165.20 & 113.80 & 447.50 & 240.40 & 138.40 & 217.10 & 190.30 \\
\hline $\operatorname{Pr}$ & 18.83 & 10.36 & 13.82 & 11.79 & 18.26 & 13.55 & 49.61 & 26.21 & 15.98 & 24.11 & 22.30 \\
\hline $\mathrm{Nd}$ & 75.00 & 39.40 & 53.70 & 49.20 & 72.80 & 56.20 & 190.80 & 103.50 & 62.70 & 91.10 & 92.00 \\
\hline $\mathrm{Sm}$ & 15.00 & 8.40 & 12.00 & 10.80 & 16.80 & 12.80 & 42.20 & 22.60 & 14.50 & 20.50 & 19.90 \\
\hline $\mathrm{Eu}$ & 0.61 & 1.14 & 1.16 & 2.93 & 1.11 & 1.26 & 4.05 & 1.56 & 1.97 & 1.40 & 2.54 \\
\hline $\mathrm{Gd}$ & 10.71 & 6.70 & 12.64 & 10.56 & 14.28 & 11.89 & 38.11 & 20.10 & 11.18 & 15.58 & 15.67 \\
\hline $\mathrm{Tb}$ & 1.66 & 1.19 & 2.28 & 1.54 & 2.54 & 2.20 & 6.06 & 3.74 & 1.94 & 2.87 & 2.64 \\
\hline Dy & 10.27 & 7.69 & 15.28 & 9.60 & 16.56 & 14.86 & 37.56 & 24.11 & 12.11 & 17.58 & 16.25 \\
\hline Ho & 2.06 & 1.52 & 3.14 & 2.06 & 3.34 & 3.01 & 7.37 & 5.14 & 2.40 & 3.63 & 3.16 \\
\hline $\mathrm{Er}$ & 6.26 & 5.07 & 8.84 & 5.49 & 10.19 & 8.82 & 21.82 & 15.46 & 6.95 & 10.67 & 9.43 \\
\hline $\mathrm{Tm}$ & 0.92 & 0.66 & 1.24 & 0.82 & 1.46 & 1.13 & 2.96 & 2.19 & 1.02 & 1.57 & 1.38 \\
\hline $\mathrm{Yb}$ & 6.62 & 4.99 & 8.19 & 5.84 & 8.92 & 7.60 & 18.51 & 14.17 & 6.71 & 10.26 & 9.47 \\
\hline $\mathrm{Lu}$ & 1.15 & 0.75 & 1.14 & 0.92 & 1.50 & 1.13 & 2.79 & 2.21 & 1.10 & 1.65 & 1.57 \\
\hline
\end{tabular}

Central European Geology 50, 2007 
Table 1 (cont.)

\begin{tabular}{|c|c|c|c|}
\hline Sample & H-8 & H-9 & H-17 \\
\hline $\mathrm{SiO}_{2}$ & 67.94 & 68.59 & 73.40 \\
\hline $\mathrm{TiO}_{2}$ & 0.33 & 0.58 & 0.17 \\
\hline $\mathrm{Al}_{2} \mathrm{O}_{3}$ & 13.63 & 12.55 & 12.87 \\
\hline $\mathrm{Fe}_{2} \mathrm{O}_{3}$ & 5.47 & 7.22 & 2.55 \\
\hline $\mathrm{MnO}$ & 0.15 & 0.06 & 0.10 \\
\hline $\mathrm{MgO}$ & 0.10 & 0.20 & 0.12 \\
\hline $\mathrm{CaO}$ & 0.92 & 0.92 & 0.69 \\
\hline $\mathrm{Na}_{2} \mathrm{O}$ & 4.91 & 3.98 & 4.10 \\
\hline $\mathrm{K}_{2} \mathrm{O}$ & 4.42 & 4.05 & 4.55 \\
\hline $\mathrm{P}_{2} \mathrm{O}_{5}$ & 0.07 & 0.06 & 0.01 \\
\hline LOI & 1.56 & 0.95 & 0.87 \\
\hline Sum & 99.50 & 99.16 & 99.43 \\
\hline
\end{tabular}

\begin{tabular}{|c|c|c|c|c|c|c|c|}
\hline \multicolumn{8}{|c|}{ Abu ad Dod AFG } \\
\hline AD-2 & AD-3 & $\begin{array}{l}\text { AD- } \\
4\end{array}$ & $\begin{array}{l}\text { AD- } \\
5 \\
\end{array}$ & $\begin{array}{l}\text { AD- } \\
6\end{array}$ & $\begin{array}{l}\text { AD- } \\
10\end{array}$ & $\begin{array}{l}\text { AD- } \\
12 \\
\end{array}$ & $\begin{array}{l}\text { AD- } \\
13\end{array}$ \\
\hline 70.03 & 72.18 & 70.24 & 66.20 & 71.85 & 69.48 & 58.74 & 62.33 \\
\hline 0.32 & 0.23 & 0.22 & 0.34 & 0.20 & 0.31 & 0.41 & 0.40 \\
\hline 10.49 & 9.46 & 11.92 & 13.61 & 10.82 & 11.25 & 14.71 & 15.54 \\
\hline 8.15 & 7.39 & 5.06 & 5.99 & 4.87 & 7.65 & 7.03 & 5.42 \\
\hline 0.27 & 0.19 & 0.11 & 0.20 & 0.13 & 0.13 & 0.27 & 0.16 \\
\hline 0.20 & 0.12 & 0.24 & 0.18 & 0.18 & 0.10 & 0.53 & 0.07 \\
\hline 0.23 & 0.22 & 1.00 & 1.04 & 1.02 & 0.66 & 2.84 & 1.41 \\
\hline 3.06 & 2.55 & 3.76 & 5.37 & 2.86 & 3.60 & 5.21 & 6.89 \\
\hline 4.94 & 4.93 & 5.04 & 4.84 & 5.56 & 5.41 & 4.98 & 5.78 \\
\hline 0.02 & 0.02 & 0.02 & 0.03 & 0.01 & 0.01 & 0.07 & 0.06 \\
\hline 1.05 & 2.44 & 2.86 & 3.66 & 2.32 & 2.04 & 4.69 & 3.04 \\
\hline 98.76 & 99.73 & 100.47 & 101.46 & 99.82 & 100.64 & 99.48 & 101.10 \\
\hline
\end{tabular}

\begin{tabular}{|c|c|c|c|}
\hline $\mathrm{Ni}$ & 2 & 2 & 1 \\
\hline $\mathrm{Co}$ & 1 & 2 & 4 \\
\hline $\mathrm{Sc}$ & 2 & 2 & 2 \\
\hline V & 3 & 3 & 3 \\
\hline $\mathrm{Cu}$ & 7 & 6 & 6 \\
\hline $\mathrm{Pb}$ & 29 & 6 & 7 \\
\hline $\mathrm{Zn}$ & 616 & 37 & 95 \\
\hline $\mathrm{Sn}$ & 11 & 17 & 10 \\
\hline W & 3 & 33 & 38 \\
\hline $\mathrm{Rb}$ & 88 & 78 & 125 \\
\hline Cs & 0.6 & 0.8 & 0.8 \\
\hline $\mathrm{Ba}$ & 409 & 203 & 131 \\
\hline $\mathrm{Sr}$ & 44 & 36 & 25 \\
\hline $\mathrm{Ga}$ & 39 & 40 & 30 \\
\hline $\mathrm{Ta}$ & 12.1 & 14.6 & 7.4 \\
\hline $\mathrm{Nb}$ & 134 & 170 & 67 \\
\hline $\mathrm{Hf}$ & 28 & 32 & 23 \\
\hline $\mathrm{Zr}$ & 962 & 1204 & 634 \\
\hline Y & 132 & 152 & 91 \\
\hline Th & 24.1 & 28.5 & 19.4 \\
\hline U & 9.3 & 7.7 & 5.6 \\
\hline $\mathrm{La}$ & 119.20 & 125.10 & 58.00 \\
\hline $\mathrm{Ce}$ & 250.00 & 287.60 & 130.40 \\
\hline $\operatorname{Pr}$ & 26.91 & 33.74 & 15.46 \\
\hline $\mathrm{Nd}$ & 103.90 & 139.20 & 59.20 \\
\hline $\mathrm{Sm}$ & 23.10 & 33.30 & 15.20 \\
\hline $\mathrm{Eu}$ & 3.43 & 3.75 & 0.60 \\
\hline $\mathrm{Gd}$ & 18.58 & 27.83 & 13.63 \\
\hline $\mathrm{Tb}$ & 3.44 & 4.70 & 2.67 \\
\hline Dy & 22.92 & 28.88 & 17.29 \\
\hline Ho & 4.86 & 5.69 & 3.58 \\
\hline $\mathrm{Er}$ & 14.62 & 17.18 & 10.42 \\
\hline $\mathrm{Tm}$ & 1.98 & 2.32 & 1.53 \\
\hline $\mathrm{Yb}$ & 13.07 & 15.63 & 9.20 \\
\hline $\mathrm{Lu}$ & 1.93 & 2.27 & 1.39 \\
\hline
\end{tabular}

\begin{tabular}{|c|c|c|c|c|c|c|c|}
\hline 1 & 2 & 2 & 1 & 3 & 1 & 10 & 1 \\
\hline 4 & 1 & 3 & 3 & 2 & 3 & 5 & 1 \\
\hline 2 & 2 & 1 & 3 & 2 & 1 & 3 & 1 \\
\hline 3 & 3 & 3 & 3 & 3 & 3 & 8 & 3 \\
\hline 7 & 11 & 6 & 12 & 5 & 4 & 7 & 31 \\
\hline 21 & 39 & 6 & 9 & 11 & 13 & 3 & 7 \\
\hline 920 & 1219 & 214 & 279 & 274 & 254 & 85 & 103 \\
\hline 38 & 50 & 9 & 14 & 16 & 12 & 5 & 7 \\
\hline 51 & 20 & 31 & 56 & 58 & 35 & 13 & 10 \\
\hline 341 & 322 & 118 & 128 & 222 & 164 & 55 & 65 \\
\hline 1.5 & 0.6 & 0.7 & 0.7 & 0.6 & 1.0 & 0.4 & 0.5 \\
\hline 152 & 190 & 95 & 624 & 112 & 142 & 621 & 505 \\
\hline 51 & 41 & 22 & 72 & 27 & 22 & 63 & 59 \\
\hline 41 & 42 & 46 & 39 & 48 & 48 & 36 & 39 \\
\hline 38.9 & 45.5 & 9.1 & 14.5 & 17.1 & 10.5 & 3.5 & 3.2 \\
\hline 446 & 884 & 103 & 184 & 180 & 125 & 55 & 53 \\
\hline 108 & 142 & 36 & 54 & 62 & 33 & 6 & 15 \\
\hline 4093 & 5617 & 1535 & 2354 & 2602 & 1466 & 228 & 734 \\
\hline 438 & 581 & 131 & 185 & 266 & 130 & 42 & 57 \\
\hline 45.9 & 89.8 & 12.2 & 15.5 & 27.2 & 13.6 & 3.8 & 5.6 \\
\hline 16.3 & 43.7 & 6.2 & 9.1 & 10.7 & 5.7 & 2.4 & 3.9 \\
\hline 459.00 & 588.20 & 126.40 & 192.50 & 200.60 & 124.70 & 64.10 & 48.40 \\
\hline 1029.00 & 1286.40 & 275.70 & 427.70 & 458.60 & 273.20 & 146.00 & 111.20 \\
\hline 110.64 & 140.84 & 31.07 & 48.90 & 52.01 & 31.02 & 17.09 & 13.22 \\
\hline 430.80 & 544.50 & 125.60 & 193.90 & 208.50 & 124.90 & 70.70 & 57.20 \\
\hline 87.00 & 103.70 & 23.90 & 37.10 & 43.00 & 23.80 & 13.80 & 11.10 \\
\hline 12.06 & 14.17 & 3.47 & 6.83 & 6.02 & 3.45 & 3.88 & 3.68 \\
\hline 80.55 & 96.57 & 23.29 & 33.00 & 42.38 & 22.49 & 11.28 & 10.61 \\
\hline 14.34 & 16.91 & 3.77 & 5.43 & 7.33 & 3.65 & 1.57 & 1.69 \\
\hline 90.72 & 109.70 & 24.52 & 32.50 & 47.54 & 23.56 & 9.13 & 10.33 \\
\hline 19.06 & 24.13 & 5.28 & 6.97 & 10.68 & 2.27 & 1.85 & 2.35 \\
\hline 53.91 & 69.30 & 15.58 & 19.16 & 30.47 & 15.66 & 4.87 & 6.63 \\
\hline 7.12 & 8.81 & 2.03 & 2.55 & 4.01 & 2.16 & 0.68 & 0.90 \\
\hline 48.98 & 60.63 & 14.16 & 17.79 & 28.99 & 15.87 & 5.17 & 6.34 \\
\hline 6.79 & 8.47 & 2.18 & 2.73 & 4.12 & 2.54 & 0.89 & 1.06 \\
\hline
\end{tabular}


270 T. M. Q adhi

Table 1 (cont.)

\begin{tabular}{|c|c|c|c|c|c|c|c|c|c|}
\hline \multicolumn{10}{|c|}{ Altered granite } \\
\hline Sample & H-3 & H-4 & H-5 & H-6 & H-7 & AD-2D & AD-7 & AD-7B & AD-8 \\
\hline $\mathrm{SiO}_{2}$ & 67.15 & 66.14 & 72.69 & 68.71 & 79.03 & 85.12 & 72.93 & 72.88 & 73.90 \\
\hline $\mathrm{TiO}_{2}$ & 0.32 & 0.38 & 0.57 & 0.73 & 0.32 & 0.11 & 0.44 & 0.56 & 0.25 \\
\hline $\mathrm{Al}_{2} \mathrm{O}_{3}$ & 14.12 & 13.46 & 5.77 & 11.83 & 3.58 & 1.60 & 6.50 & 6.73 & 8.71 \\
\hline $\mathrm{Fe}_{2} \mathrm{O}_{3}$ & 5.14 & 6.79 & 9.66 & 8.28 & 8.87 & 7.47 & 11.39 & 9.93 & 7.38 \\
\hline $\mathrm{MnO}$ & 0.24 & 0.11 & 0.63 & 0.09 & 0.38 & 0.19 & 0.11 & 0.06 & 0.12 \\
\hline $\mathrm{MgO}$ & 0.69 & 0.13 & 0.07 & 0.25 & 0.04 & 0.39 & 0.28 & 0.24 & 0.13 \\
\hline $\mathrm{CaO}$ & 1.35 & 1.42 & 0.37 & 0.53 & 0.14 & 0.37 & 0.30 & 0.21 & 0.44 \\
\hline $\mathrm{Na}_{2} \mathrm{O}$ & 5.45 & 3.81 & 0.15 & 3.62 & 0.12 & 0.18 & 1.09 & 0.81 & 1.60 \\
\hline $\mathrm{K}_{2} \mathrm{O}$ & 2.68 & 4.80 & 2.54 & 3.79 & 1.86 & 0.39 & 3.25 & 4.90 & 6.02 \\
\hline $\mathrm{P}_{2} \mathrm{O}_{5}$ & 0.07 & 0.03 & 0.26 & 0.10 & 0.10 & 0.11 & 0.02 & 0.02 & 0.01 \\
\hline LOI & 3.22 & 2.12 & 7.25 & 3.06 & 6.11 & 4.17 & 4.03 & 3.65 & 2.86 \\
\hline Sum & 100.43 & 99.19 & 99.96 & 100.99 & 100.55 & 100.10 & 100.34 & 99.99 & 101.42 \\
\hline $\mathrm{Ni}$ & 7 & 1 & 2 & 4 & 6 & 3 & 2 & 1 & 2 \\
\hline Co & 10 & 2 & 4 & 5 & 4 & 7 & 1 & 3 & 1 \\
\hline $\mathrm{Sc}$ & 6 & 2 & 9 & 3 & 6 & 3 & 2 & 2 & 1 \\
\hline V & 22 & 3 & 11 & 3 & 21 & 7 & 3 & 3 & 3 \\
\hline $\mathrm{Cu}$ & 38 & 8 & 100 & 13 & 13 & 29 & 14 & 33 & 11 \\
\hline $\mathrm{Pb}$ & 22 & 24 & 92 & 8 & 62 & 35 & 31 & 30 & 9 \\
\hline $\mathrm{Zn}$ & 293 & 723 & 1951 & 46 & 1390 & 1106 & 800 & 343 & 256 \\
\hline $\mathrm{Sn}$ & 6 & 34 & 226 & 23 & 201 & 71 & 127 & 157 & 35 \\
\hline W & 19 & 24 & 69 & 49 & 109 & 133 & 19 & 43 & 29 \\
\hline $\mathrm{Rb}$ & 55 & 175 & 194 & 74 & 135 & 24 & 284 & 345 & 345 \\
\hline Cs & 0.9 & 0.5 & 1.4 & 0.8 & 1.0 & 1.8 & 0.4 & 0.5 & 0.6 \\
\hline $\mathrm{Ba}$ & 356 & 208 & 390 & 202 & 251 & 107 & 262 & 271 & 96 \\
\hline $\mathrm{Sr}$ & 107 & 30 & 73 & 57 & 25 & 28 & 35 & 52 & 29 \\
\hline $\mathrm{Ga}$ & 32 & 40 & 45 & 37 & 31 & 14 & 36 & 35 & 45 \\
\hline $\mathrm{Ta}$ & 6.3 & 23.8 & 194.3 & 17.7 & 143.2 & 60.1 & 48.6 & 50.8 & 15.7 \\
\hline $\mathrm{Nb}$ & 51 & 280 & 2043 & 195 & 3483 & 1978 & 587 & 849 & 340 \\
\hline $\mathrm{Hf}$ & 13 & 57 & 288 & 47 & 174 & 50 & 310 & 368 & 79 \\
\hline $\mathrm{Zr}$ & 394 & 1910 & 12598 & 2142 & 7713 & 2847 & 12746 & 14887 & 3273 \\
\hline Y & 82 & 308 & 2166 & 178 & 1338 & 714 & 565 & 430 & 156 \\
\hline Th & 15.7 & 33.9 & 571.6 & 26.4 & 534.9 & 255.6 & 45.9 & 156.9 & 47.3 \\
\hline $\mathrm{U}$ & 3.9 & 15.4 & 142.8 & 10.5 & 95.7 & 76.9 & 55.4 & 92.3 & 15.9 \\
\hline $\mathrm{La}$ & 70.50 & 222.40 & 3115.70 & 149.10 & 1884.70 & 1175.10 & 593.00 & 532.70 & 340.10 \\
\hline $\mathrm{Ce}$ & 177.90 & 495.60 & 6697.80 & 315.40 & 3541.00 & 2043.00 & 1247.30 & 1006.20 & 572.10 \\
\hline Pr & 18.09 & 55.46 & 795.17 & 36.44 & 414.86 & 206.39 & 135.31 & 104.77 & 55.77 \\
\hline $\mathrm{Nd}$ & 71.70 & 218.60 & 2973.60 & 148.30 & 1519.50 & 747.50 & 511.00 & 384.20 & 195.60 \\
\hline $\mathrm{Sm}$ & 16.30 & 47.60 & 568.40 & 33.60 & 298.50 & 122.90 & 98.30 & 68.70 & 31.20 \\
\hline $\mathrm{Eu}$ & 1.76 & 5.45 & 68.68 & 3.88 & 35.44 & 15.98 & 13.17 & 9.29 & 3.99 \\
\hline $\mathrm{Gd}$ & 13.47 & 44.71 & 462.04 & 29.69 & 237.76 & 102.36 & 92.43 & 65.12 & 26.14 \\
\hline $\mathrm{Tb}$ & 2.46 & 7.99 & 66.64 & 5.02 & 36.23 & 16.59 & 15.78 & 10.62 & 3.99 \\
\hline Dy & 15.82 & 53.72 & 378.10 & 31.79 & 210.66 & 102.15 & 104.14 & 68.00 & 24.00 \\
\hline Ho & 3.11 & 11.25 & 70.06 & 6.65 & 39.63 & 19.47 & 22.05 & 14.20 & 4.76 \\
\hline $\mathrm{Er}$ & 9.26 & 35.70 & 197.46 & 20.00 & 110.94 & 46.77 & 60.23 & 40.58 & 13.42 \\
\hline $\mathrm{Tm}$ & 1.27 & 4.92 & 24.07 & 2.80 & 13.80 & 5.02 & 7.52 & 5.17 & 1.79 \\
\hline $\mathrm{Yb}$ & 8.43 & 31.57 & 142.20 & 18.16 & 38.06 & 30.92 & 51.65 & 37.53 & 13.37 \\
\hline $\mathrm{Lu}$ & 1.38 & 4.81 & 19.02 & 2.78 & 11.02 & 3.64 & 7.19 & 5.29 & 2.08 \\
\hline
\end{tabular}

Central European Geology 50, 2007 


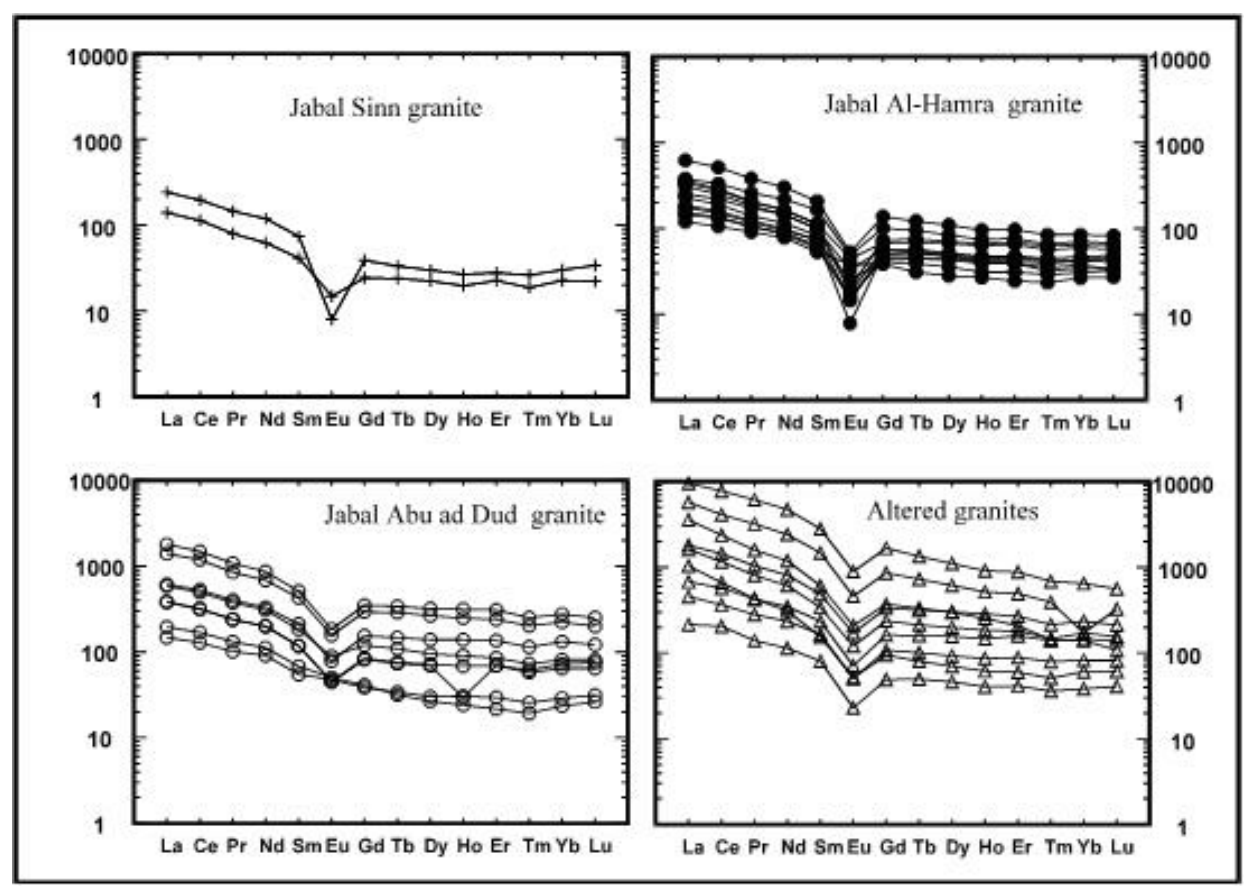

Fig. 6

Primitive mantle-normalized multi-element diagrams for the investigated granites. Normalizing values are from Sun and McDonough (1989)

acterized by a smooth negative slope with LREE enrichment (Lan = 39-204), fractionated patterns ( $\mathrm{La} / \mathrm{Ybn}=4-6 ; \mathrm{La} / \mathrm{Smn}=2.3-3.2)$ and moderate to strong negative Eu anomalies $\left\{\left(\mathrm{Eu} / \mathrm{Eu}^{*}=0.22-0.84\right)\right\}$. Compared to the rocks of Jabal Hamra (Table 1), the Abu ad Dud alkali feldspar granite shows regular fractionated patterns, similar to the Jabal Hamra rocks $(\mathrm{La} / \mathrm{Ybn}=4.6-8 ; \mathrm{La} / \mathrm{Smn}=$ 2.7-3.5) but with moderate negative Eu-anomalies $\left\{\left(\mathrm{Eu} / \mathrm{Eu}^{*}=0.36-1\right)\right\}$ and higher total REE ( $\Sigma$ REE $=325-3072 \mathrm{ppm})$. The close similarity of REE patterns of the Jabal Hamra and Jabal Abu ad Dud rocks, and the variation in Eu anomalies and total REE, indicate that all the rocks are presumably developed from the same magma source, but the Hamra granites show a more fractionated nature. The syenogranite of Jabal Sinn shows fractionated REE patterns ( $\mathrm{La} / \mathrm{Ybn}=6.2$ and $\mathrm{La} / \mathrm{Smn}=1.1)$ and negative Eu anomalies $\left\{\left(\mathrm{Eu} / \mathrm{Eu}^{*}=0.46\right)\right\}$ similar to the Hamra and Abu ad Dud rocks, but with lower total REE (average $\Sigma$ REE $=232$ ppm).

The hydrothermally altered granites show high and wide ranges in REE enrichment ( $\left(a_{n} 200\right.$ up to 10,000; and $Y b_{n}=13$ to 142) and have more fractionated REE patterns than their host or parent rock (quart alkali feldspar syenite), but with similar negative Eu anomalies $\left\{\mathrm{La} / \mathrm{Ybn}=4.7-25.4\right.$, $\left(\mathrm{Eu} / \mathrm{Eu}^{*}=0.36\right.$ - 


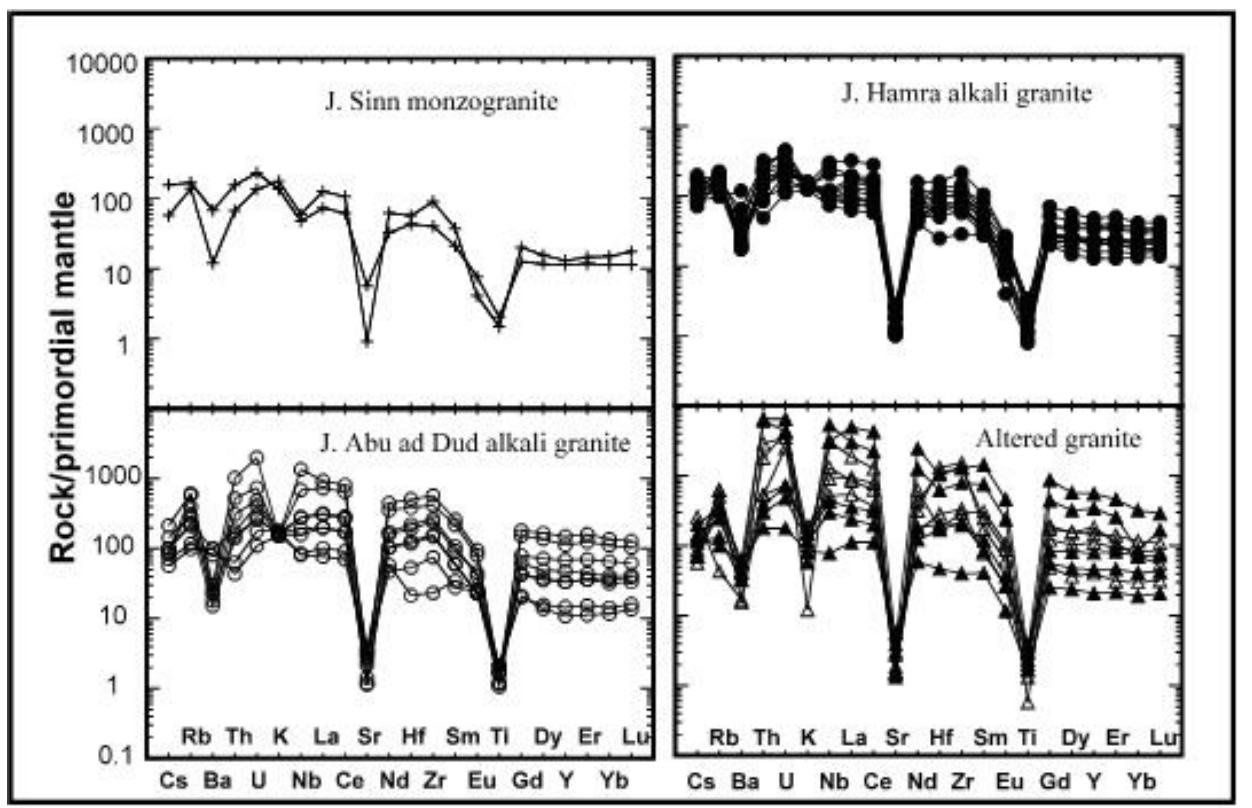

Fig. 7

Chondrite-normalized REE patterns of the investigated granites. Normalizing value are from Sun (1982)

$0.44)\}$. The most striking features of this type of hydrothermal alteration is the anomalous enrichment in the total REE ( $\Sigma$ REE $=804-15579$ ppm).

\section{Granite type and tectonic setting}

The type and tectonic setting of the investigated granites are determined mainly for the unaltered granites. The granites of Jabal Hamra and Abu ad Dud are all hypersolvous and metaluminous to marginally peralkaline. They have a pronounced depletion in $\mathrm{CaO}, \mathrm{MgO}, \mathrm{Sr}, \mathrm{Ba}$, and a marked enrichment in $\mathrm{FeO} * / \mathrm{MgO}, \mathrm{Ga} / \mathrm{Al}, \mathrm{Rb} / \mathrm{Sr}$, HFSE (Zr, Y, Nb, Ta, Hf, Zn) and REE. These chemical aspects closely resemble the A-type granites of both orogenic- and anorogenic silicic magmatism in the sense of many workers (Whalen et al. 1987; Chappell et al. 1987; Eby 1990, 1992; Whalen et al. 1996). The binary scatter diagrams $Y$ versus $\mathrm{Nb}$ (Pearce et al. 1984) and $(\mathrm{Zr}+\mathrm{Nb}+\mathrm{Y}+\mathrm{Ce})$ versus $\mathrm{FeO} * / \mathrm{MgO}$ (Whalen et al. 1987) are commonly used to discriminate the A-type granites from the other types (I- and S-type granites). All the investigated granites fall entirely in the Atype granitic fields (Fig. 8a, b). Eby (1990-1992) used the abundance of $\mathrm{Ce}, \mathrm{Y}$ and $\mathrm{Nb}$ to differentiate between orogenic ( $\mathrm{A}_{1}$-subtype) and anorogenic granites $\left(\mathrm{A}_{2}-\right.$ subtype) associated with rift zone. On the $\mathrm{N} \mathrm{b}-\mathrm{Ce}-\mathrm{Y}$ diagram (Fig. 8c) the Hamra and Abu ad Dud alkali feldspar granite and alkali feldspar syenite samples cluster 
around the dividing line and plot in both $A_{1}$-subtype and $A_{2}$-subtype, but most of the samples lie in the postcollision $\mathrm{A}_{2}$-subtype granite field.

\section{Sr and $\mathrm{N} \mathrm{d}$ isotopes}

The Jabal Hamra quartz alkali feldspar syenite has been dated in this study by the whole-rock Rb-Sr method. The analytical results are given in Table (2) and plotted in Fig. 9. Five samples of the Jabal Hamra quartz alkali feldspar syenite were selected to obtain a wide spread in $\mathrm{Rb} / \mathrm{Sr}$ ratios; the analytical points define a moderate straight-line fit on a ${ }^{87} \mathrm{Sr} /{ }^{86} \mathrm{Sr}$ versus ${ }^{87} \mathrm{Rb} /{ }^{86} \mathrm{Sr}$ isochron diagram (Fig. 9). The "isochron age" calculated from the five analytical points is $538 \pm 15$ Ma with an initial ${ }^{87} \mathrm{Sr} /{ }^{86} \mathrm{Sr}$ ratio of $0.7068 \pm 0.001(2 \sigma)$. The observed scatter of points about the "errorchron" exceeds the analytical uncertainty (MSWD = 19) and could be due to a late open system behavior induced by post-magmatic alteration. Although the rocks of the Hamra pluton are locally affected by variable hydrothermal alteration, the samples selected for isotopic measurements show the minimal effect of this type of alteration. N evertheless,
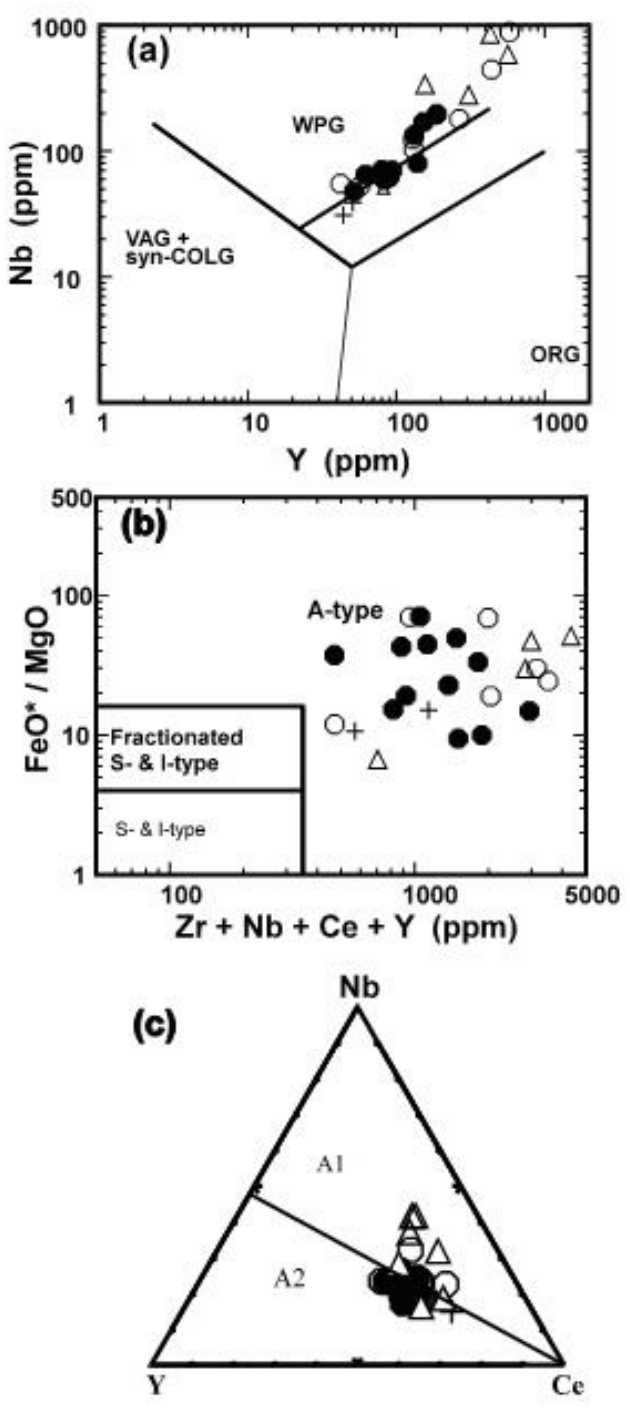

Fig. 8

Tectonic discrimination diagrams for the investigated granites. a) Y vs. Nb diagram (Pearce et al. 1984), VAG volcanic arc granite, syn-COLG - syn-collision granite, ORG - ocean ridge granite, WPG - within plate granite; $b$ ) $(\mathrm{FeO} * / \mathrm{MgO}$ vs. $\mathrm{Zr}+\mathrm{Nb}+\mathrm{Ce}+\mathrm{Y}$ discrimination diagram (Whalen et al. 1987); c) Y-N b-Ceternary diagram (Eby 1992) $\mathrm{A} 1$ and $\mathrm{A} 2$ are the fields of anorogenic and post-orogenic granites, respectively. Symbols as in Fig. 3 


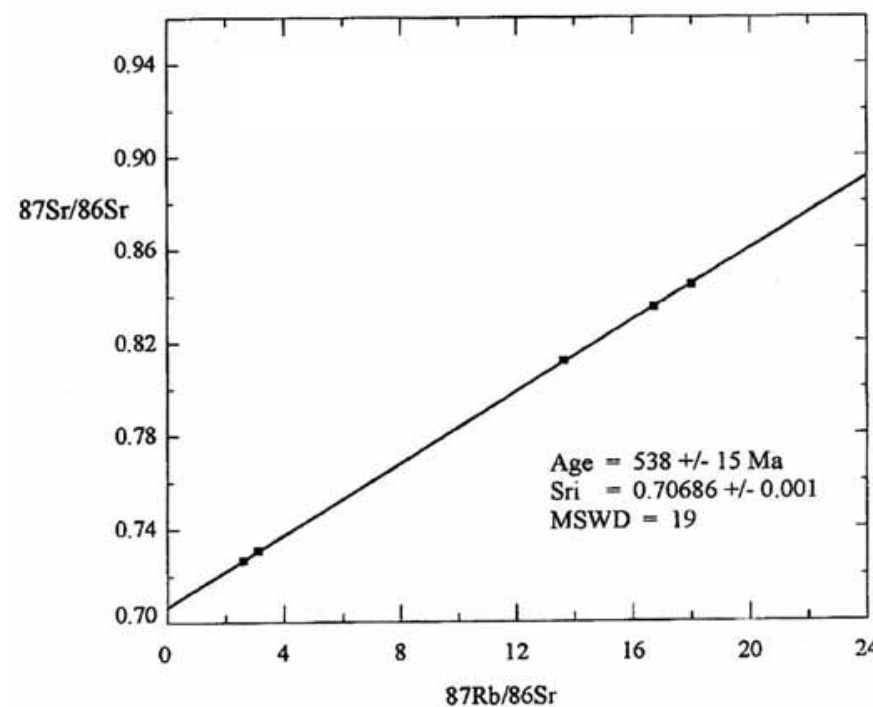

Fig. 9

$\mathrm{Rb}-\mathrm{Sr}$ whole rock isochron for the Jabal Al Hamra alkali granite

incipient alteration of feldspar and biotite cannot be completely excluded. The age $(538 \mathrm{Ma})$ and the moderate initial ${ }^{87} \mathrm{Sr} /{ }^{86} \mathrm{Sr}$ ratio $(0.7068)$ are in good agreement with other ages $(590-510 \mathrm{Ma})$ and moderately high initial $87 \mathrm{Sr} / 86 \mathrm{Sr}$ ratios (0.705-0.714) reported for many alkaline/peralkaline types of granite from the Arabian Shield (Duyverman et al. 1982; Calvez and Kemp 1982; Calvez et al. 1983; Q adhi 2003). The moderately high initial ${ }^{87} \mathrm{Sr} /{ }^{86} \mathrm{Sr}$ ratio of the Jabal Hamra rocks indicates that other sources (e.g. crustal materials or hydrothermal fluids) may be involved in their evolution.

Neodiumum isotopic compositions were determined for four petrographically selected samples from the Hamra pluton (quartz alkali feldspar syenite) and one sample (\# H-20) of syenogranite from Jabal Sinn. The data and some calculated parameters, including $\mathrm{Nd}$-model ages (TDM) and eNd values, are presented in Table 2. The Hamra rocks are characterized by positive eNd values $(+3.52-$ +4.23), which are comparable to the REE-bearing A-type granites in the Arabian Shield. The similar eNd values and the close chemical similarity (e.g. LILE and HFS elements) between Jabal Hamra and Jabal Sinn indicate that they are genetically-related and both were derived from, at least, a chemically and isotopically similar magma source. The wholly positive values of the eNd indicate a source region which has a high $\mathrm{Sm} / \mathrm{Nd}$ ratio; such a source is normally interpreted as a mantle or a juvenile crustal source (e.g. Faure 1986). The isotopic composition shows that the Hamra syenite and Jabal Sinn syenogranite have positive $\varepsilon \mathrm{Nd}$ and $\varepsilon \mathrm{Sr}$ values and thus a "mantle" $\mathrm{Nd}$ isotopic signature and a "crustal" Sr isotopic signature. However, the $\mathrm{N}$ d values of the Hamra syeniteand Jabal Sinn syenogranite lie within the $\varepsilon N d$ range $(+2.7$ to 5.1$)$ reported for Arabian Shield continental crust (Duyverman et al. 1982; Stacey and Hedge 1984; 
Table 2

$\mathrm{Rb}-\mathrm{Sr}$ and $\mathrm{Sm}-\mathrm{Nd}$ data for Al Hamra granites

\begin{tabular}{cccccccc}
\hline $\begin{array}{c}\text { Sample } \\
\text { No. }\end{array}$ & ${ }^{87} \mathrm{Rb} /{ }^{86} \mathrm{Sr}$ & ${ }^{87} \mathrm{Sr} /{ }^{86} \mathrm{Sr}( \pm 2 \sigma)$ & ${ }^{147} \mathrm{Sm} /{ }^{144} \mathrm{Nd}$ & $\begin{array}{c}{ }^{143} \mathrm{Nd} /{ }^{144} \mathrm{Nd} \\
( \pm 2 \sigma)\end{array}$ & $\varepsilon^{\mathrm{t}} \mathrm{Sr}_{\mathrm{r}}$ & $\varepsilon^{\mathrm{t}} \mathrm{Nd}$ & $\mathrm{T}_{\mathrm{DM}}(\mathrm{GA})$ \\
\hline H-2 & 3.10 & $0.731117 \pm 7$ & 0.130 & $0.512629 \pm 6$ & 49 & 4.23 & 0.68 \\
$\mathrm{H}-11$ & 13.62 & $0.811933 \pm 8$ & 0.131 & $0.512596 \pm 6$ & 51 & 3.52 & 0.74 \\
H-17 & 18.00 & $0.844256 \pm 9$ & 0.136 & $0.512653 \pm 6$ & 33 & 4.29 & 0.68 \\
H-18 & 16.72 & $0.834720 \pm 10$ & & & 37 & & 0.69 \\
H-20 & 2.60 & $0.726371 \pm 9$ & 0.119 & $0.512588 \pm 4$ & 36 & 4.19 & 0.67 \\
\cline { 2 - 7 }
\end{tabular}

The $2 \sigma$ standard error in ${ }^{87} \mathrm{Rb} /{ }^{86} \mathrm{Sr}$ and ${ }^{147} \mathrm{Sm} /{ }^{144} \mathrm{Nd}$ is $1 \%$. Analytical uncertainties in ${ }^{87} \mathrm{Sr} /{ }^{86} \mathrm{Sr}$ used to weigh the regression and calculate the MSWD are $0.2 \%$

Hegner and Pallister 1989; McGuire and Stern 1993; Frost et al. 2001). The calculated $\mathrm{Nd}$-depleted mantle model ages (TDM) for the investigated Hamra syenite and Jabal Sinn syenogranite range from 0.67 to $0.74 \mathrm{Ga}(0.70 \mathrm{Ga}$ on average) and are higher than the calculated crystallization age. This may imply that the mantle source of the studied rocks is less depleted than that of the depleted mantle from which the $\mathrm{Nd}$ model ages were calculated.

\section{Discussion}

Petrogenesis of the A-type granites

To explain the origin of A-type granites, a number of petrogenetic models have been proposed in which crustal, mantle-derived or mixed sources were suggested. Numerous $\mathrm{Sm}-\mathrm{Nd}$ and $\mathrm{Rb}-\mathrm{Sr}$ isotope data obtained during the last decade suggest that many silicic magmas of this type were produced from sources in which mantle-derived material was dominant (e.g. Turner et al. 1992; Whalen et al. 1996; Jahn et al. 2000; Bonin 2004; Jahn et al. 2004). The isotope data on the investigated granites support this conclusion. Positive eNd $(T)$ values ranging from +3.5 to +4.2 in the investigated granites (Table 2) point to a 'juvenile' character and suggest that the silicic magma was derived either by partial melting of juvenile crust or by differentiation of mantle-derived magma. It follows from data in Table 2 that the crystallization age $(538 \mathrm{Ma})$ and $\mathrm{N} \mathrm{d}$ model age TDM (670-740 Ma) differ significantly. Since TDM corresponds to the time when the source of granite magma has been isolated from the mantle source (De Paolo et al. 1991) the observed difference suggests later thermal reworking of the mantle source material and possibly admixture of crustal component. This means that the eNd $(T)$ values calculated for granites cannot be interpreted straightforwardly as an indication of mildly depleted mantle source of granite (Arndt and Goldstein 1987). However, these values attest that the granites from the Hamra area contain more than $50 \%$ mantle (or young juvenile crust) component. 
Similar models were suggested for other A-type granites of the Arabian-Nubian Shield (Beyth et al. 1994; M oghazi 1999; Katzir et al. 2006). The high initial Sr ratio (0.7068) may be attributed to addition of Sr from hydrothermal fluids.

Fractional crystallization, generally of a mantle-derived magma, to produce Atype granites or peralkaline compositions has been proposed by many workers (e.g. Turner et al. 1992). One argument against a mechanism of fractional crystallization of a mafic magma to produce the A-type granites of the study area is that some of the least evolved rocks have $\mathrm{Eu}, \mathrm{Rb}, \mathrm{Sr}$ and $\mathrm{Ba}$ contents that preclude extensive feldspar fractionation from a mafic source. The composition of the investigated rocks [Ba up to $750 \mathrm{ppm}$; Rb, up to $340 \mathrm{ppm}$; and low to moderate $\mathrm{Eu}$, up to $4 \mathrm{ppm}$ ] indicates that there has been no appreciable feldspar fractionation. The most mafic members of the investigated granites $(\mathrm{H}-2$ and $\mathrm{H}-14$, Table 1) have compositions that were probably not produced by fractionation from a mafic source, but appear to be the result of partial melting. Magma mixing is a process that has been advanced by some workers to produce A-type granites. H owever, as many of the A-type granites of the studied area are peralkaline, very little strongly metaluminous mafic magma could have been added to the granite magma, especially considering that the mafic magmas have higher concentrations of Al.

Many workers have suggested that A-type granites are produced by combined partial melting-fractionation from source regions of slightly different composition than those for I-type granites (e.g. Collins et al. 1982; Clemens et al. 1986; Whalen et al. 1987; Creaser et al. 1991; Landenberger and Collins 1996). Proposed source compositions that have been favored are lower crust, including tonalite, granodiorite, peraluminous granulite, charnockite and granulitic residuum from melting of I-type granites. We favor a felsic mantle-derived crustal source that could overlap in composition with I-type sources. The high temperatures required to produce an extractable magma may have been initiated by mantle upwelling or mafic magma influx into a localized area. The generated melt evolved by fractional crystallization of feldspars, ferromagnesian minerals and some accessory phases to produce the petrographic variation in the studied granites.

$\mathrm{H}$ ydrothermal alteration and mineralization

Petrographic study reveals that most of the Jabal Hamra and Jabal Abu ad Dud rocks preserve little-modified magmatic relationships. The texture of both alkali feldspar granite and alkali feldspar syenite is hypidiomorphic with subhedral to euhedral grains of quartz, plagioclase, alkali feldspar and biotite. Alkali feldspar is variably perthitic, and film perthite is more widely developed than flame and bleb types. Rare grains of apparently uniform, unexsolved alkali feldspar are observed. The igneous mica varies from dark red-brown to orangey brown and encloses accessory phases, including apatite, zircon and monazite. However, 
many of the biotite crystals are altered to chlorite-iron oxide mixture. Several studies showed that abundance of exsolution textures in alkali feldspar indicates extensive structural rearrangement of magmatic alkali feldspar at low $\left(=400{ }^{\circ} \mathrm{C}\right)$ temperature (e.g. Parsons 1978; Brown and Parsons 1989; Worden et al. 1990; Lee et al. 1995; Walker et al. 1995). Theturbidity in alkali feldspars points to recrystallization driven by infiltration of aqueous fluid. According to Parsons (1978), coarse patch perthite in subsolvus feldspars developed isochemically by water-impelled dissolution and reprecipitation. This suggests that in the course of recrystallization the system remained closed. Closed-system behavior is also supported by the small scatter in Rb- Sr isochron constructed for the Hamra samples. Thus the $\mathrm{Rb}-\mathrm{Sr}$ isotope system was not disturbed by sub-solidus interaction with fluids. It follows that the main proportion of water that caused the feldspar recrystallization should come from the granite magma itself. The dominance of exsolution and alteration textures of perthite grains from the alkali feldspar granites suggests that fluid phase was present for a long time after the emplacement and crystallization of the plutons.

The dominant alteration process in the granitoid rocks hosting the rare-metal mineralization was the replacement of perthitic orthoclase by kaoline and sericite with a remarkable change in megascopic and microscopic appearance. With increasing alteration turbidity extends throughout the grains, with concomitant development of abundant iron oxides (hematite dust) along the grain boundaries. In the most strongly metasomatized rocks, pitted textures become pervasive within $\mathrm{K}$ feldspar grains. Moreover, $\mathrm{Na-metasomatism}$ is erratically distributed and characteristically takes the form of replacive rims of white albite on the margins of the alkali feldspar megacrysts, although groundmass plagioclase is also albitized. From the standpoint of hydrothermal alteration the rare-metalbearing altered granite should be considered as a product of intense hydrothermal-metasomatic alteration of the alkali feldspar granite and alkali feldspar syenite. This is defined by: 1) position in the field, where gradational contact between the altered and non-altered granite is recorded, 2) different modal composition, 3) chemical composition characterized by higher $\mathrm{TiO}_{2}, \mathrm{Fe}_{2} \mathrm{O}_{3}, \mathrm{SiO}_{2}$ and lower $\mathrm{Al}_{2} \mathrm{O}_{3}, \mathrm{CaO}, \mathrm{Na}_{2} \mathrm{O}$, than for the unaltered rocks and 4) extraordinarily high concentrations of HFSE and REE.

\section{O rigin of raremetal mineralization}

High contents of HFSE in the alkali feldspar granite and alkali feldspar syenite can be attributed to characteristics of the source region and to those of the corresponding melts, which allow them to accommodate higher proportions of these elements than other melts (Scarfe 1977). The elevated contents of alkalis and fluorine in these magmas, as indicated by modal fluorite and high $\mathrm{Na}_{2} \mathrm{O}+\mathrm{K}_{2} \mathrm{O}$ in these rocks, increase the solubility of HFSE by promoting formation of polymeric alkali-silicate complexes and/or alkali-fluoride complexes 
with these metals (Watson 1979; Collerson 1982; Collins et al. 1982). The effects of fluorine in granites is well known and it is suggested that the F-rich fluid could produce HREE and HFSE enrichment in the late stages of evolution of granitic melt due to F complexing (Dingwell 1988; Rogers and Satterfield 1994). The addition of $\mathrm{F}$ into the melt promotes the formation of $\left[\mathrm{SiO}_{3} \mathrm{~F}\right]^{3-}$ complexes of HREE and HFSE, resulting in depolymerization and reduction in the availability of $\mathrm{SiO}_{4}$ tetrahedra to form HREE- and HFSE-bearing phases (Dingwell 1988). The development of F-bearing fluids and their interaction with the silicic melt at the late magmatic stage enhance element fractionation, since the addition of fluorine decreases silicate melt viscosity (Mysen and Virgo 1985) and expands the primary phase field of quartz (Manning 1981; Manning and Pichavant 1983), thus extending the duration of crystallization. However, the altered granite bodies contain extraordinarily high concentrations of HFSE that igneous processes al one seem insufficient to account for. A feature of plutons showing extreme enrichment in these elements is an association of mineralization with hydrothermal alteration (Trueman et al. 1988), which suggests that hydrothermal processes played a role in HFSE concentration.

In a study of mass changes during alteration, Salvi and Williams-Jones (1996) have been able to show quantitatively that appreciable quantities of $\mathrm{Zr}, \mathrm{Y}$ and heavy REE were concentrated hydrothermally. This enrichment was explained by orthomagmatic fluid transport of these elements as fluoride complexes, and their subsequent deposition as a result of mixing with an externally derived $\mathrm{Ca}$ and/or Ferich meteoric fluid. The Hamra and Abu ad Dud plutons present alteration patterns very similar to those associated with the hydrothermal raremetal mineralization of Salvi and Williams-Jones (1996); it is therefore reasonable to speculate that similar additions of $\mathrm{Zr}, \mathrm{Y}$ and heavy REE occurred in this complex. The hydrothermal solutions may have originated form the igneous intrusions and have undergone mixing with other fluids rich in $\mathrm{Fe}, \mathrm{MnO}, \mathrm{TiO}_{2}$ and $\mathrm{Zn}$. The Hamra area is therefore an ideal locality to test the hypothesis formulated for rare-metal mineralization formed by hydrothermal fluids in the Arabian Shield. Further fluid inclusion and stable isotope studies are required to evaluate this process.

\section{Conclusions}

1. The Hamra granite plutons, emplaced in a Neoproterozoic island arc assemblage in northeast Arabia, are an example of alkaline felsic magmatism that host rare-metal mineralization. The granites consist of three granitic types, alkali feldspar granite, alkali feldspar syenite and altered granite. The alkali feldspar granite and alkali feldspar syenite satisfy the mineralogical, geochemical (major and trace elements) and tectonic requirements of post-collision A-type granite.

2. The alkali-feldspar granite and syenite were generated mainly by fractional crystallization of crustal-derived magma. Abundance of turbid alkali feldspars 
and altered biotite suggest that crystallization of alkali-feldspar granites was followed by sub-solidus fluid-rock interaction. High eN d $(T)$ values $(+3.5$ and +4.2 ) indicate that the granite magma was generated from a 'juvenile' source, which is typical of the rocks making up most of the Arabian-Nubian shield.

3. Rare metal mineralization associated with fracture-controlled alteration zones occurs at Jabal Al Hamra and Jabal Abu ad Dud. The altered rocks are characterized by higher $\mathrm{TiO}_{2}, \mathrm{Fe}_{2} \mathrm{O}_{3}, \mathrm{SiO}_{2}$, and lower $\mathrm{Al}_{2} \mathrm{O}_{3}, \mathrm{CaO}, \mathrm{Na}_{2} \mathrm{O}$, than the unaltered rocks. They show a high and wide range in the total REE contents (804-15579 ppm), Ta (6-194 ppm), Nb (51-3483 ppm), Hf (13-368 ppm), Zr (394-14887 ppm), Th (16-572 ppm) and U (4-143 ppm).

4. Field observations and further petrographic and chemical studies suggest that the altered rocks and the rare metal enrichment are the products of hydrothermal-metasomatic alteration of the quartz alkali feldspar syenite and the alkali feldspar granite. The rare-metal enrichment is explained by orthomagmatic fluid transport of these elements as fluoride complexes, and their subsequent deposition as a result of mixing with an externally derived Ferich fluid.

\section{R eferences}

Arndt, N.T., I.V. Goldstein 1987: Use and abuse of crust-formation ages. - Geology, 15, pp. 893-895. Batchelor, R.A., P. Bowden 1985: Petrogenetic interpretation of granitoid rock series using multicationic parameters. - Chem. Geol., 48, pp. 43-55.

Beyth, M., R.J. Stern, R. Altherr, A. Kroner 1994: The late Precambrian Timna igneous complex, southern Israel: Evidence for comagmatic-type sanukitoid monzodiorite and alkali granite magma. - Lithos, 31, pp. 103-124.

Bonin, B. 2004: Do coeval mafic and felsic magmas in postcollisional to within-plate regimes necessarily imply two contrasting, mantle and crustal, sources? A review. - Lithos, 78, pp. 1-24.

Brown, W.L., I. Parsons 1989: Alkali feldspars: ordering rates, phase transformations and behavior diagrams for igneous rocks. - Mineral. Mag., 53, pp. 25-42.

Calvez, J.Y., J. Kemp 1982: Geochronological investigations in the Mahd adh Dhahab Quadrangle, central Arabian Shield. - Saudi Arabian Deputy Ministry of Mineral Resources Techn. Rec. BRGM-TR-02-5, $40 \mathrm{p}$.

Calvez, J.Y., C. Alsac, J. Delfour, J. Kemp, C. Pellaton 1983: Geologic evolution of western, central, and eastern parts of the northern Precambrian shield, Kingdom of Saudi Arabia. - Saudi Arabian Deputy Ministry for Mineral Resources Open-File Report BRGM-OF-02-26, 39 p.

Chappell, W.B., A.J. White, D. Wyborn 1987: The importance of residual source material (restite) in granite petrogenesis. - J. Petrol., 28, pp. 1111-1138.

Clemens, J.D., J.R. Holloway, A.J.R. White 1986: Origin of an A-type granite: experimental constraints. - Am. Miner., 79, pp. 71-86.

Collerson, K.D. 1982: Geochemistry and Rb-Sr geochronology of associated Proterozoic peralkaline and subalkaline anorogenic granites from Labrador. - Contrib. Mineral. Petrol., 81, pp. 126-147.

Collins, W.J., S.D. Beams, A.J.R. White, B.W. Chappell 1982: Nature and origin of A-type granites with particular reference to southeastern Australia. - Contrib. Mineral. Petrol., 80, pp. 189-200.

Creaser, R.A., R.C. Price, R.J. Wormald 1991: A-type granite revisited: assessment of residual source model. - Geology, 19, pp. 163-166.

De La Roche, H., J. Leterrier, P. Grandelaude, M. Marchal 1980: A classification of volcanic and plutonic rocks using R1R2-diagram and major-element analyses - its relationships with current nomenclature. - Chem. Geol., 29, pp. 183-210. 
DePaolo, D.J. 1981: Trace element and isotopic effects of combined wallrock assimilation and fractional crystallization. - Earth Planet. Sci. Lett., 53, pp. 189-202.

De Paolo, D.J., A.M. Linn, G. Schubert 1991: The continental crust age distribution methods to determining mantle separation ages from $\mathrm{Sm}-\mathrm{Nd}$ isotopic data and application to the southwestern United States. - J. Geophys. Res., 96, pp. 2071-2088.

Dingwell, D.B. 1988: The structures and properties of fluorine-rich magmas: A review of experimental studies. - In Taylor, R.P., D.F. Strong (Eds): Recent advances in the study of granite-related mineral deposits. - Can. Instit. Mineral. Metallog., Montreal Quebec, pp. 1-12.

Drysdall, A.R., N.J. Jackson, C.R. Ramsay, C.J. Douch, A.D. Hackett 1984: Rare element mineralization related to Precambrian alkali granites in the northwest Arabian Shield. - Econ. Geol., 79, pp. 1366-1377.

Du Bray, E.A. 1986: Specialized granitoids in the southeastern Arabian Shield - case history of a regional assessment. - J. Afr. Earth Sci., 4, pp. 169-176.

Duyverman, H.J., N.B.W. Harris, C.J. Hawkesworth 1982: Crustal accretion in the Pan-African: Nd and $\mathrm{Sr}$ isotope evidence from the Arabian Shield. - Earth Planet. Sci. Lett., 59, pp. 315-326.

Eby, G.N. 1990: The A-type granitoids: a review of their occurrence and chemical characteristics and speculations on their petrogenesis. - Lithos, 26, pp. 115-134.

Eby, G.N. 1992: Chemical subdivisions of the A-type granitoids: petrogenesis and tectonic implications. - Geology, 20, pp. 641-644.

Faure, G. 1986: Principles of Isotope Geology. - (2nd Ed.). John Wiley and Sons, New York, 586 p.

Fleck, R.J., W.R. Greenwood, D.G. Hadley, R.E. Anderson, D.L. Schmidt 1980: Age and evolution of the southern part of the Arabian shield, in Al-Shanti, A.M., convenor, Evolution and mineralization of the Arabian Nubian shield. - King Abdulaziz University, Institute of Applied Geology Bulletin 3, 4, pp. 1-17 (Pergamon Press, Oxford).

Frost, B.R., C.G. Barnes, W.J. Collins, R.J. Arculus, D.J. Ellis, C.D. Frost 2001: A geochemical classification for granitic rocks. - J. Petrol., 42, pp. 2033-2048.

Greenwood, W.R., D.G. Hadley, R.E. Anderson, R.J. Fleck, D.L Schmidt 1976: Late Proterozoic cratonization in southwestern Saudi Arabia. - Philos. Transact. Royal Soc. London, Series A 280, pp. 517-527.

Hadley, D.G. 1975: Geology of the Qalat Sawrah quadrangle, sheet 26/38 D, Kingdom of Saudi Arabia. - Saudi Arabian Directorate General of Mineral Resources Geologic Map GM-24, scale $1: 100,000$.

Hadley, D.G. 1987: Geologic map of the Sahl al Matran quadrangle, sheet 26 C Kingdom of Saudi Arabia. - Saudi Arabian Directorate General of Mineral Resources. Geologic Map GM-86 C, scale 1:250,000.

Hegner, E., J.S. Pallister 1989: Pb, Sr, Nd isotopic characteristics of Tertiary Red sea rift volcanics from the central Saudi Arabian coastal plain. - Am. Geophys. Union, pp. 7749-7755.

Jackson, N.J. 1986: Mineralization associated with felsic plutonic rocks in the Arabian Shield. - J. Afr. Earth Sci., 4, pp. 213-227.

Jackson, N.J., C.J. Douch 1986: Jabal Hamra REE-mineralized silexite, Hijaz region, Kingdom of Saudi Arabia. - J. Afr. Earth Sci., 4, pp. 269-274.

Jahn, B.-M., R. Capdevila, D. Liu, A. Vernov, G. Badarch 2004: Sources of Phanerozoic granitoids in the transect Bayanhongor-Ulan Baator, Mongolia: geochemical and $\mathrm{Nd}$ isotopic evidence, and implications of Phanerozoic crustal growth. - J. Asian Earth Sci., 23, pp. 629-653.

Jahn, B.-M.,F.Y. Wu, B. Chen 2000: Granitoids of the Central Asian Orogenic Belt and continental growth in the Phanerozoic. - Trans. R. Soc. Edinb. Earth Sci., 91, pp. 181-193.

Katzir, Y., M. Eyal, B.A. Litvinovsky, B.M. Jahn, A.N. Zanvilevich, J.W. Valley, Y. Beeri, I. Pelly, E. Shimshilashvili 2006: Petrogenesis of A-type granites and origin of vertical zoning in the Katharina pluton, Gebel Mussa (Mt. Moses) area, Sinai, Egypt. - Lithos, 95/3-4, pp. 208-228.

Kemp, J., C. Pellaton, J.Y. Calvez 1980: Geochronological investigations and geologic history in the Precambrian of north-western Saudi Arabia. - Saudi Arabian Deputy Ministry for Mineral Resources Open-File Report BRGM-OF-01-1, 120 p. 
Landenberger, B., W.J. Collins 1996: Derivation of A-type granites from dehydrated charnockitic lower crust: evidence from the Chaelundi complex Eastern Australia. - J. Petrol., 37, pp. $145-170$.

Lee, M.R., K.A. Waldron, I. Parsons 1995: Exsolution and alteration microtextures in alkali feldspar phenocrysts from the Shap granite. - Mineral. Mag., 59, pp. 63-78.

Maniar, D.P., P.M. Piccoli 1989: Tectonic discrimination of granitoids. - GSA Bull., 101/5, pp. 635-643.

Manning, D.A.C. 1981: The effect of fluorine on liquidus phase relationships in the system Qz-AbOr with excess water. - Contrib. Mineral. Petrol., 76, pp. 206-215.

Manning, D.A.C., M. Pichavant 1983: The role of fluorine and boron in the generation of granitic rocks. - In: Atherton, M.P., C.D. Gribble (Eds): Migmatites, melting and metamorphism. - Shiva Nantwich., pp. 94-110.

McGuire, A.V., R.J. Stern 1993: Granulite xenoliths from western Saudi Arabia: the lower crust of the late Precambrian Arabian-Nubian Shield. - Contrib. Mineral. Petrol., 114, pp. 395-408.

Moghazi, A.M. 1999: Magma source and evolution of Late N eoproterozoic granitoids in the Gabal El-Urf area, Eastern Desert, Egypt: geochemical and Sr-Nd isotopic constraints. - Geol. Mag., 136, pp. 285-300.

Mysen, B.O., D. Virgo 1985: Structure and properties of fluorine-bearing alumosilicate melts: the system $\mathrm{Na}_{2} \mathrm{O}-\mathrm{Al}_{2} \mathrm{O}_{3}-\mathrm{SiO}_{2}-\mathrm{F}$ at 1 atm. - Contrib. Mineral. Petrol., 91, pp. 205-220.

Parsons, I. 1978: Feldspars and fluids in cooling plutons.- Mineral. Mag., 42, pp. 1-17.

Pearce, J.A., N.B.W. Harris, A.G. Tindle 1984: Trace element discrimination diagrams for the tectonic interpretation of granitic rocks. - J. Petrol., 25, pp. 956-983.

Qadhi, T.M. 1990: Petrogenesis and mineralization of the Ghurayyah pluton, Midyan, Northeast Saudi Arabia. - Ph.D. Thesis Unpublished. King Abdulaziz University, Jeddah.

Qadhi, T. 2003: Rare-metal bearing peralkaline granite in Um Al Birak area, Arabian Shield, KSA. Geochemical and Sr-Nd isotope study (in press).

Ramsay, C.R. 1986: Specialized felsic plutonic rocks of the Arabian Shield and their precursors. J. Afr. Earth Sci., 4, pp. 153-168.

Ramsay, C.R., A.R. Drysdall, M.D. Clark 1986: Felsic plutonic rocks of the Midyan region, Kingdom of Saudi Arabia - I. Distribution, classification and resource potential. - J. Afr. Earth Sci., 4, pp. 63-77.

Rogers J.J.W., M.E. Satterfield 1994: Fluids of anorogenic granites: A preliminary assessment. Mineral. Petrol., 50, pp. 157-171.

Salvi, S., A.E. Williams-Jones 1996: The role of hydrothermal processes in concentrating HFSE in the Strange Lake peralkaline complex, northeastern Canada. - Geochim. Cosmochim. Acta, 60, pp. 1917-1932.

Scarfe, C.M. 1977: Viscosity of a pantellerite melt at one atmosphere. - Can. Mineral., 15, pp. 185-189.

Schmidt, D.L., D.G. Hadley, D.B. Stoeser 1979: Late Proterozoic crustal history of the Arabian Shield southern Najd Province, kingdom of Saudi Arabia. - Instit. Appl. Geol. Bull., 3, pp. 41-58.

Schwartz, M.O. 1992: Geochemical criteria for distinguishing magmatic and metasomatic albiteenrichment in granitoids - examples from the Ta-Li granite Yichun (China) and the Sn-W deposit Tikus (Indonesia). - Mineral. Deposita, 27, pp. 101-108.

Stacey, J.S., C.E. Hedge 1984: Geochronologic and isotopic evidence for early Proterozoic crust in the eastern Arabian shield. - Geology, 12, pp. 310-313.

Steiger, R.H., E. Jager 1977: Subcommission on geochronology; the use of decay constants in geoand cosmochronology. - Earth and Planet. Sci. Lett., 36, pp. 359-362.

Stern, R.J. 1994: Arc assembly and continental collision in the Neoproterozoic East African Orogeny: implications for the consolidation of Gondwanaland. - Ann. Rev. Earth Planet. Sci., 22, pp. 319-351.

Stern, R.J. 2002: Crustal evolution in the East African Orogen: a neodymium isotopic perspective. J. Afr. Earth Sci., 34, pp. 109- 117. 
Stoeser, D.B. 1986: Distribution and tectonic setting of plutonic rocks of the Arabian Shield. - J. Afr. Earth Sci., 4. pp. 21-46.

Sun, S-S. 1982: Chemical composition and origin of the Earth's primitive mantle. - Geochim. Cosmochim. Acta, 46, pp. 179-192.

Sun, S-S., W. F. McDonough 1989: Chemical and isotopic systematics of oceanic basalts: implications for mantle composition and processes. - Geological Society, London, Spec. Publ., 42, pp. 313-345.

Tischendorf, G. 1977: Geochemical and petrographic characteristics of silicic magmatic rocks associated with rare-element mineralization. - In: Metallization associated with acid magmatism 2, pp. 41-96. Czechoslovakian Geologic Survey, Prague.

Trueman, D.L., J.C. Pedersen, L. de St. Jorre, D.G.W. Smith 1988: The Thor Lake rare-metal deposits, Northwest Territories. - Canadian Institute of Mining and Metallurgy Spec. Vol. 39, pp. 279-284.

Turner, S.P., J.D. Foden, R.S. Morrison 1992: Derivation of some A-type granites by fractionation of basaltic magma: an example from the Padthaway Ridge, South Australia. - Lithos, 28, pp. 51-179.

Walker, F.D.L., M.R. Lee, I. Parsons 1995: Micropores and micropermeable texture in alkali feldspar: geochemical and geophysical implications. - Mineral. Mag., 59, pp. 505-534.

Watson, E.B. 1979: Zircon saturation in felsic liquids: Experimental results and applications to trace elements geochemistry. - Contrib. Mineral. Petrol, 70, pp. 407-419.

Whalen, J.B., K.L. Currie, B.W. Chappell 1987: A-type granites: geochemical characteristics, discrimination and petrogenesis. - Contrib. Mineral. Petrol, 95, pp. 407-419.

Whalen, J.B., G.A. Jenner, F.J. Longstaffe, F. Robert, C. Gariépy 1996: Geochemical and isotopic (O, $\mathrm{Nd}, \mathrm{Pb}$ and $\mathrm{Sr}$ ) constraints on A-type granite petrogenesis based on the Topsails Igneous Suite Newfoundland Appalachians. - J. Petrol., 37, pp. 1463-1489.

Worden, R.H., F.D.L. Walker, W.L. Brown. 1990: Development of microporosity, diffusion channels and deuteric coarsening in perthitic alkali feldspars. - Contrib. Mineral. Petrol., 104, pp. 507-515. 